\title{
CLIENT ACCEPTANCE METHOD FOR AUDIT FIRMS BASED ON INTERVAL-VALUED FUZZY NUMBERS
}

\author{
Hung-Lin LAI ${ }^{\mathrm{a}}$, Ting-Yu CHEN ${ }^{\mathrm{b}}$ \\ ${ }^{a}$ Department of Accounting Information, Chihlee Institute of Technology, 313, Sec. 1, \\ Wun-Hua Road, Banciao District, 22050 New Taipei City, Taiwan \\ ${ }^{\mathrm{b}}$ Department of Industrial and Business Management, \\ Graduate Institute of Business and Management, College of Management, \\ Chang Gung University, 259, Wen-Hwa 1st Road, \\ Kwei-Shan, 333 Taoyuan, Taiwan
}

Received 18 February 2012; accepted 28 July 2012

\begin{abstract}
To ensure that investors are getting financial statements that conform to Generally Accepted Accounting Principles, the security exchange committee requires publicly traded companies to hire external auditors. Because the information provided in company financial statements has significant economic and social consequences for various parties, external auditors are needed to minimize litigation. Therefore, it is important for audit firms' risk management teams to evaluate which clients to accept. In this paper, we propose a client acceptance method (CAM) that uses a technique for order preference using similarity to the ideal solution (i.e. TOPSIS) approach to evaluate potential new clients using a decision-making method with interval-valued fuzzy numbers (IVFNs). Through a case study, this paper shows that this CAM results in a high Spearman rankorder correlation coefficient ( 0.9 to 1.0 ) with human judgment. This result indicates that CAM could help decision makers evaluate potential clients before acceptance, especially when there are several potential clients but limited resources to provide services. The CAM also could help audit firms more easily ensure that decision makers are complying with firm policies concerning client acceptance through the establishment of uncertainty factor weights.
\end{abstract}

Keywords: risk management, client acceptance, TOPSIS, decision making, interval-valued fuzzy numbers.

JEL Classification: C44, D81, G28, N55, O16.

Corresponding author Ting-Yu Chen

E-mail: tychen@mail.cgu.edu.tw 


\section{Introduction}

Investors often rely on information provided in audited financial statements when making decisions about investment in a company's securities (Schneider 2011). Therefore, two key features of the financial reporting system are that an audit is mandated for publicly traded companies, and an audit must be conducted by accountants who are independent in fact as well as in appearance (Jamal, Sunder 2011). From a theoretical perspective, the need for auditing can be explained by Stewardship Theory and Agency Theory (Joshi et al. 2009). Agency theory, as described by Jensen and Meckling in 1976, states that credible financial reporting reduces the asymmetry of information between corporate managers and stockholders, improves investor confidence and raises the stock price, which makes it less costly for corporations to raise new equity capital for growth. Stewardship theory states that managers, with no oversight, will act as responsible stewards of the assets they control. Agency theory provides the theoretical basis for hiring auditors to provide independent assurance to investors that a firm's financial statements conform to Generally Accepted Accounting Principles (GAAP) and are free from material error.

For every audit, the audit staff carries the audit risk of possibly submitting inaccurate opinions and the responsibility to avoid such errors (Chang et al. 2008). Auditors need to be knowledgeable about, and understanding of, relevant problems arising from their clients' ever-changing business circumstances (Joshi et al. 2009). Even when auditors exercise appropriate scrutiny and present proper audit opinions, they may still risk lawsuits if the auditee's business fails (Chang et al. 2008). Litigation risk can influence the decision-making process of auditors during the audit (Hwang, Chang 2010). Business failure results when an enterprise cannot pay off its debts or satisfy its investors' expectations because of the external economic environment or its internal corporate circumstances. When auditors do not use accepted audit practices according to the appropriate audit criteria and submit wrong audit opinions, an audit failure will result (Chang et al. 2008).

Once a new client has been accepted, audit firms generally face problems related to obtaining the required information, meeting the deadline date, auditing multiple large clients at the same time, and understanding the operations of new clients (Joshi et al. 2009). An audit firm's work load will be heavy if it accepts many new clients simultaneously. Moreover, audit firms experience conflict between the business of auditing and the profession of auditing, which is manifested in high pressure to perform quality work within specified time limits at the audit manager and senior levels (Joshi et al. 2009).

Craig (1992) argued that procedures should be established by auditing firms to guide them when deciding which audit engagements to accept and which to decline. Because information provided by companies has significant economic and social consequences for various parties, external auditors are needed to minimize litigation (Moizer, Smith 1998). Audit firms should evaluate any unusual risks inherent in a potential audit engagement (Joshi et al. 2009). Research has shown that engagement risk influences decision-making behavior and is an important aspect of the general audit environment (e.g. Johnson et al. 2002). Therefore, deciding which clients to accept is an important issue for audit firms' risk management. 
This paper is organized as follows. In Section 1, we briefly present a literature review on issues related to the proposed method, such as risk management, fuzzy sets, and the technique for order preference by similarity to the ideal solution (TOPSIS). In Section 2, we briefly review the basic concepts of IVFNs (Chen 2006; Wang, Li 1998) and some existing similarity measures between IVFNs. In Section 3, we propose a new method to analyze new audit client acceptance problems between IVFNs using a TOPSIS approach combined with a similarity measure. In Section 4, we apply the proposed method to an audit firm in the client-acceptance process using a case study. The conclusions are presented in the final section.

\section{Literature review}

Much of the existing risk management research has been based on the use of linguistic assessments instead of numerical values. For example, Idrus et al. (2011) used linguistic terms to analyze project risk assessment. Lai and Chen (2011) applied linguistic assessment in the accounting decision-making process. Chen and Lai (2011) also applied linguistic terms in risk assessment for enhancing patient safety. The fuzzy set is a mathematical tool for the analysis of data defined in imprecise linguistic terms based on subjective judgments, such as low-risk, serious-impact, or high-probability events. It has been widely applied to human decision-making processes. For example, Zhang and Liu (2010) used triangular intuitionistic fuzzy numbers to select one person from four candidates to be a department manager.

When something is uncertain, such as a measurement, using type- 1 fuzzy sets that represent uncertainty with numbers in the range $[0,1]$ makes more sense than using conventional sets. This method has been applied widely for decision making, even in the medical system. Steimann (2001) investigated the impact that fuzzy set theory has had on work in medical artificial intelligence and outlines the strengths of using fuzzy set theory. However, it may not be reasonable to use an accurate membership function for something that is not only uncertain but also complex (Sepulveda et al. 2007). The concept of type-2 fuzzy sets has therefore been proposed (Zadeh 1975), and this concept may better handle linguistic uncertainties in complex situations. A type-2 fuzzy set can be defined by a fuzzy membership function, the grade (or fuzzy grade) of which is taken to be a fuzzy set in the unit interval $[0,1]$ rather than a specific point within $[0,1]$ (Mizumoto, Tanaka 1981).

$\mathrm{Xu}$ and Yager (2008) explained that in many complex decision-making problems, the information provided by a decision maker is often imprecise or uncertain due to time pressure, lack of data, or the decision maker's limited attention and information processing capabilities. The interval-valued fuzzy numbers (IVFNs) were defined from type-2 fuzzy sets by Zadeh (1975) and Sambuc (1975) and have been popularly adopted for handling uncertainties arising from incomplete or imprecise information. For example, Zhai and Mendel (2011) generated generalized definitions to such type- 2 fuzzy sets and created a unified strategy for computing all different uncertainty measures with a low complexity; Chen (2011) presented a new method to reduce cognitive dissonance and to relate optimism and pessimism in multiple criteria decision analysis in an interval-valued fuzzy decision environment; and Leal-Ramirez et al. (2011) proposed an age-structured population growth model, based on a fuzzy cellular structure. Chen (2012) provided a group decision-making method under incomplete preference 
information via signed distances in the context of generalized interval-valued trapezoidal fuzzy numbers. Baležentis and Zeng (2013) extended the MULTIMOORA method with type-2 fuzzy sets viz. generalized interval-valued trapezoidal fuzzy numbers. Farhadinia (2014) presented the sensitivity analysis in interval-valued trapezoidal fuzzy number linear programming problems.

Some researchers have applied fuzzy sets to risk management. For example, excluding research mentioned in first paragraph, Schmucker (1984) elaborated on fuzzy sets, natural language computations and risk analysis in his book; García et al. (1992) obtained fuzzy sets that are as close as possible to the natural expression of systems needing an estimative calculation of risk, whether it is the risk of failure, access or of any other type, with incomplete and/ or imprecise data; Han and Liu (2011) also provided a fuzzy multi-attribute, decision-making method under the risk framework with unknown attribute weights; Cimpoeru (2011) applied fuzzy sets in credit risk assessment; Wei et al. (2013) investigated the multiple attribute group decision-making (MAGDM) problems in which the attribute values take the form of triangular fuzzy information; Yu (2013) applied multi-criteria group decision making based on intuitionistic fuzzy prioritized operators under intuitionistic fuzzy environment; and Wu et al. (2013), Tseng and Chiu (2013), Govindan et al. (2013), and Chang and Yeh (2013) also applied fuzzy sets in supply chain management. IVFNs may also be applied to address risk management problems. Chen, S. J. and Chen, S. M. (2008) and Wei and Chen (2009) proposed methods to address risk management problems based on IVFNs. Because uncertainty is an inherent attribute of information (Zadeh 2005), these methods would appear to be an applicable method for audit firms to evaluate the variable and uncertain factors in the client acceptance process.

According to fuzzy set theory, it is often difficult for an expert to identify their opinion as a number in the interval $[0,1]$. Therefore, representing the degree of certainty of an opinion in an interval is more appropriate for real-world circumstances. The use of intervals is a characteristic of IVFNs. For the reasons mentioned above, we evaluated the potential new clients of audit firms using IVFNs in this study. Simultaneously, we use the technique for order preference by similarity to the ideal solution (TOPSIS) approach developed by Hwang and Yoon (1981), a widely used multiple-attribute, decision-making method. TOPSIS considers the distances to both the ideal and the negative ideal solutions simultaneously by finding the relative closeness to the ideal solution. There are five steps in the TOPSIS approach. First, a normalized decision matrix is constructed. Second, a weighted normalized decision matrix is constructed. Then, ideal and negative ideal solutions are determined. After calculating the separation measure, the relative closeness to the ideal solution is calculated. Finally, the preference order is ranked.

The basic concept behind TOPSIS is that the chosen alternative should have the shortest possible distance from the positive-ideal solution and the farthest possible distance from the negative-ideal solution. To evaluate potential new clients, we use TOPSIS to compare them with the positive-ideal solution and the negative-ideal solution. Rather than measuring the distance between the alternatives and the positive/negative ideal solution, we measure the similarity between the IVFNs of the scores of potential new clients and the positive ideal solution/negative ideal solution, which can lead to more intuitive results than measuring the distance. 


\section{Preliminaries of IVFNs and similarity measures}

\subsection{Preliminaries of IVFNs}

Wang and Li (1998) defined IVFNs and gave them extended operations. From Chen (2006), the IVFN $\tilde{\tilde{A}}$, shown in Figure 1 , can be represented by $\tilde{\tilde{A}}=\left[\tilde{\tilde{A}}^{\mathrm{L}}, \tilde{\tilde{A}}^{\mathrm{U}}\right]$, where $\tilde{\tilde{A}}^{\mathrm{L}}$ denotes the lower IVFN and $\tilde{\tilde{A}}^{\mathrm{U}}$ denotes the upper IVFN and $\tilde{\tilde{A}}^{\mathrm{L}} \subset \tilde{\tilde{A}}^{\mathrm{U}}$.

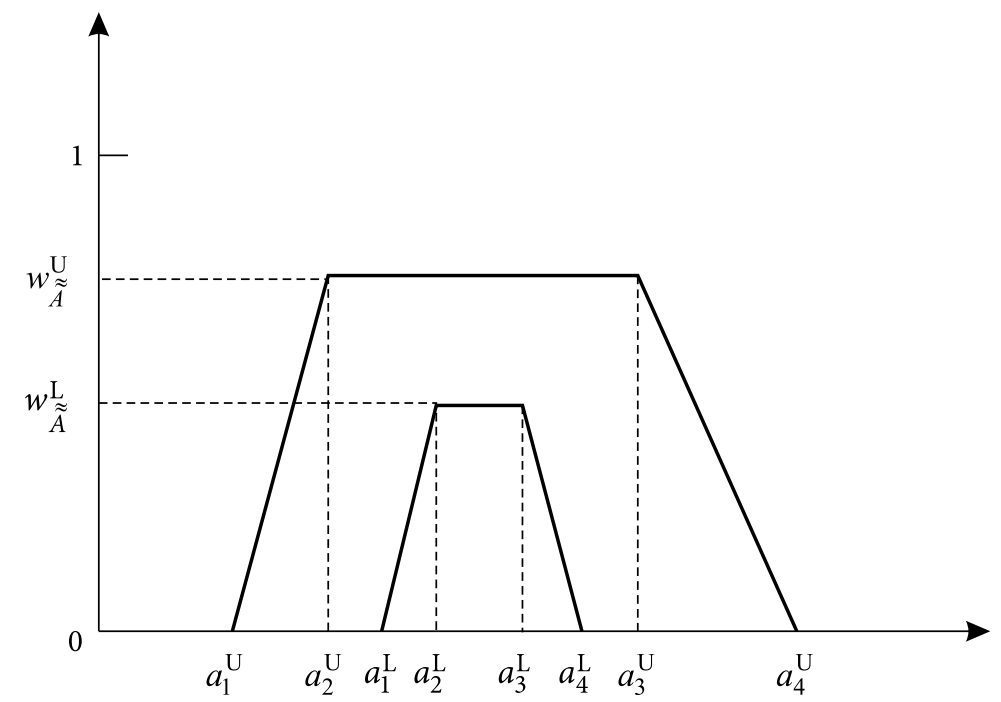

Fig. 1. An interval-valued trapezoidal fuzzy number

Let $\tilde{\tilde{A}}$ and $\tilde{\tilde{B}}$ be two IVFNs, where $\tilde{\tilde{A}}=\left[\tilde{\tilde{A}}^{\mathrm{L}}, \tilde{\tilde{A}}^{\mathrm{U}}\right]=\left[\left(a_{1}^{\mathrm{L}}, a_{2}^{\mathrm{L}}, a_{3}^{\mathrm{L}}, a_{4}^{\mathrm{L}} ; w_{\tilde{\tilde{A}}}^{\mathrm{L}}\right)\right.$, $\left.\left(a_{1}^{\mathrm{U}}, a_{2}^{\mathrm{U}}, a_{3}^{\mathrm{U}}, a_{4}^{\mathrm{U}} ; w_{\tilde{\tilde{A}}}^{\mathrm{U}}\right)\right], \quad \tilde{\tilde{B}}=\left(\tilde{\tilde{B}}^{\mathrm{L}}, \tilde{\tilde{B}}^{\mathrm{U}}\right)=\left[\left(b_{1}^{\mathrm{L}}, b_{2}^{\mathrm{L}}, b_{3}^{\mathrm{L}}, b_{4}^{\mathrm{L}} ; w_{\tilde{\tilde{B}}}^{\mathrm{L}}\right),\left(b_{1}^{\mathrm{U}}, b_{2}^{\mathrm{U}}, b_{3}^{\mathrm{U}}, b_{4}^{\mathrm{U}} ; w_{\tilde{\tilde{B}}}^{\mathrm{U}}\right)\right]$, $0 \leq w_{\tilde{\tilde{A}}}^{\mathrm{L}} \leq w_{\tilde{\tilde{A}}}^{\mathrm{U}} \leq 1, \quad \tilde{\tilde{A}}^{\mathrm{L}} \subset \tilde{\tilde{A}}^{\mathrm{U}}, \quad 0 \leq w_{\tilde{\tilde{B}}}^{\mathrm{L}} \leq w_{\tilde{\tilde{B}}}^{\mathrm{U}} \leq 1 \quad$ and $\quad \tilde{\tilde{B}}^{\mathrm{L}} \subset \tilde{\tilde{B}}^{\mathrm{U}}$. The arithmetic operations between IVFNs $\tilde{\tilde{A}}$ and $\tilde{\tilde{B}}$ were presented in the research by Chen in 2006 .

\subsection{Similarity measures between IVFNs}

Several methods for measuring the similarity of fuzzy numbers have been presented in the fuzzy theory literature. Pappis and Karacapilidis (1995) presented a grading system for the similarity of two fuzzy sets and defined its properties. Wu and Mendel (2009) analyzed five existing similarity measures for interval type-2 fuzzy sets and proposed a similarity measure with reduced computational complexity. Zhang, H. Y. and Zhang, W. X. (2009) introduced a new definition of an inclusion measure, the hybrid monotonic inclusion measure. Chen, S. J. and Chen, S. M. (2008) proposed a similarity measure for calculating the degree of similarity between IVFNs using geometric concepts to calculate the center-of-gravity (COG) points of the lower and upper fuzzy numbers of IVFNs. 
Chen, S. J. and Chen, S. M. (2004) proposed a method for calculating the degree of similarity between IVFNs based on COG points. Here, the COG-based similarity measure was used to calculate the degree of similarity $S\left(\tilde{\tilde{A}}^{\mathrm{L}}, \tilde{\tilde{B}}^{\mathrm{L}}\right)$ between the lower trapezoidal fuzzy numbers $\tilde{\tilde{A}}^{\mathrm{L}}$ and $\tilde{\tilde{B}}^{\mathrm{L}}$, and the degree of similarity $S\left(\tilde{\tilde{A}}^{\mathrm{U}}, \tilde{\tilde{B}}^{\mathrm{U}}\right)$ between the upper trapezoidal fuzzy numbers $\tilde{\tilde{A}}^{\mathrm{U}}$ and $\tilde{\tilde{B}}^{\mathrm{U}}$ of the two IVFNs $\tilde{\tilde{A}}$ and $\tilde{\tilde{B}}$. However, the COG-based similarity measure used by Chen, S. J. and Chen, S. M. (2004) cannot correctly handle the similarity measure of two different generalized fuzzy numbers that have the same COG points. Therefore, Chen (2006) proposed a fuzzy-number similarity measure to overcome this drawback and to present a new method for calculating the degree of similarity between IVFNs. Wei and Chen (2009) presented a similarity measure between IVFNs that combined the concepts of the geometric distance, the perimeter, the height and the COG points of IVFNs to calculate the degree of similarity between IVFNs.

Chen and Sanguansat (2011) presented a similarity measure between IVFNs that considered the degrees of closeness between IVFNs on the X-axis and the degrees of differences between the shapes of the IVFNs on the X-axis and the Y-axis. They also proved three properties of the proposed similarity measure. Chen and Sanguansat (2011) used nine sets of IVFNs from Wei and Chen (2009) to compare their results with those of some existing methods. The comparison showed that Chen and Sanguansat's method and Wei and Chen's method yielded more reasonable results than other methods. They also used six sets of IVFNs from Chen, S. J. and Chen, S. M. (2009) to compare their results with those of several existing methods. The results also showed that Chen and Sanguansat's method and Chen and Chen's method yielded more reasonable results than other methods, indicating that Chen and Sanguansat's (2011) method can overcome the drawbacks of the methods developed earlier. Below, we briefly describe the similarity measure method presented by Chen and Sanguansat (2011).

Let $\tilde{\tilde{A}}$ and $\tilde{\tilde{B}}$ be two IVFNs as described in Section 1, the degree of similarity $S(\tilde{\tilde{A}}, \tilde{\tilde{B}})$ between IVFNs $\tilde{\tilde{A}}$ and $\tilde{\tilde{B}}$ is calculated as follows:

$$
S(\tilde{\tilde{A}}, \tilde{\tilde{B}})=D C\left(\tilde{\tilde{A}}^{\mathrm{U}}, \tilde{\tilde{B}}^{\mathrm{U}}\right) \times\left(1-D D_{X}(\tilde{\tilde{A}}, \tilde{\tilde{B}})\right) \times\left(1-D D_{Y}(\tilde{\tilde{A}}, \tilde{\tilde{B}})\right)
$$

and $S(\tilde{\tilde{A}}, \tilde{\tilde{B}}) \in[0,1]$.

First, the degree of closeness $D C\left(\tilde{\tilde{\tilde{A}}}^{\mathrm{U}}, \tilde{\tilde{B}}^{\mathrm{U}}\right)$ between the upper-trapezoidal fuzzy numbers $\tilde{\tilde{A}}^{\mathrm{U}}$ and $\tilde{\tilde{B}}^{\mathrm{U}}$ of the interval-valued fuzzy numbers $\tilde{\tilde{A}}$ and $\tilde{B}$ on the $\mathrm{X}$-axis are calculated:

$$
D C\left(\tilde{\tilde{A}}^{\mathrm{U}}, \tilde{\tilde{B}}^{\mathrm{U}}\right)=1-\frac{\left|\sum_{i=1}^{4}\left(a_{i}^{\mathrm{U}}-b_{i}^{\mathrm{U}}\right)\right|}{4} .
$$

Next, an array of differences between the corresponding values of the IVFNs $\tilde{\tilde{A}}$ and $\tilde{\tilde{B}}$ on the $\mathrm{X}$-axis and an array of differences between the membership degrees of the corresponding values of the IVFNs $\tilde{\tilde{A}}$ and $\tilde{\tilde{B}}$ are calculated, respectively:

$$
\begin{aligned}
P= & {\left[x_{1}, x_{2}, x_{3}, x_{4}, x_{5}, x_{6}, x_{7}, x_{8}\right]=} \\
& {\left[a_{1}^{\mathrm{L}}-b_{1}^{\mathrm{L}}, a_{2}^{\mathrm{L}}-b_{2}^{\mathrm{L}}, a_{3}^{\mathrm{L}}-b_{3}^{\mathrm{L}}, a_{4}^{\mathrm{L}}-b_{4}^{\mathrm{L}}, a_{1}^{\mathrm{U}}-b_{1}^{\mathrm{U}}, a_{2}^{\mathrm{U}}-b_{2}^{\mathrm{U}}, a_{3}^{\mathrm{U}}-b_{3}^{\mathrm{U}}, a_{4}^{\mathrm{U}}-b_{4}^{\mathrm{U}}\right] ; }
\end{aligned}
$$




$$
\begin{aligned}
Q= & {\left[y_{1}, y_{2}, y_{3}, y_{4}, y_{5}, y_{6}, y_{7}, y_{8}\right]=} \\
{[} & f_{\tilde{\tilde{A}}^{\mathrm{L}}}\left(a_{1}^{\mathrm{L}}\right)-f_{\tilde{\tilde{B}}^{\mathrm{L}}}\left(b_{1}^{\mathrm{L}}\right), f_{\tilde{\tilde{A}}^{\mathrm{L}}}\left(a_{2}^{\mathrm{L}}\right)-f_{\tilde{\tilde{B}}^{\mathrm{L}}}\left(b_{2}^{\mathrm{L}}\right), f_{\tilde{\tilde{A}}^{\mathrm{L}}}\left(a_{3}^{\mathrm{L}}\right)-f_{\tilde{\tilde{B}}^{\mathrm{L}}}\left(b_{3}^{\mathrm{L}}\right), \\
& f_{\tilde{\tilde{A}}^{\mathrm{L}}}\left(a_{4}^{\mathrm{L}}\right)-f_{\tilde{\tilde{B}}^{\mathrm{L}}}\left(b_{4}^{\mathrm{L}}\right), f_{\tilde{\tilde{A}}^{\mathrm{U}}}\left(a_{1}^{\mathrm{U}}\right)-f_{\tilde{\tilde{B}}^{\mathrm{U}}}\left(b_{1}^{\mathrm{U}}\right), f_{\tilde{\tilde{A}}^{\mathrm{U}}}\left(a_{2}^{\mathrm{U}}\right)-f_{\tilde{\tilde{B}}^{\mathrm{U}}}\left(b_{2}^{\mathrm{U}}\right), \\
& \left.f_{\tilde{\tilde{A}}^{\mathrm{U}}}\left(a_{3}^{\mathrm{U}}\right)-f_{\tilde{\tilde{B}}^{\mathrm{U}}}\left(b_{3}^{\mathrm{U}}\right), f_{\tilde{\tilde{A}}^{\mathrm{U}}}\left(a_{4}^{\mathrm{U}}\right)-f_{\tilde{\tilde{B}}^{\mathrm{U}}}\left(b_{4}^{\mathrm{U}}\right)\right],
\end{aligned}
$$

where the definition of each component can be found in Chen and Sanguansat (2011).

The mean of elements in the two arrays mentioned above have also been calculated as follows:

$$
\begin{aligned}
& \bar{X}=\frac{\sum_{i=1}^{8} x_{i}}{8} ; \\
& \bar{Y}=\frac{\sum_{i=1}^{8} y_{i}}{8} .
\end{aligned}
$$

Then, the $\underset{\tilde{A}}{\operatorname{An}}$ degrees of the difference $D D_{X}(\tilde{\tilde{A}}, \tilde{\tilde{B}})$ and $D D_{Y}(\tilde{\tilde{A}}, \tilde{\tilde{B}})$ between the shapes of the IVFNs $\tilde{\tilde{A}}$ and $\tilde{\tilde{B}}$ on the $\mathrm{X}$-axis and Y-axis, respectively, are calculated.

$$
\begin{aligned}
& D_{X}(\tilde{\tilde{A}}, \tilde{\tilde{B}})=\sqrt{\frac{\sum_{i=1}^{8}\left(x_{i}-\bar{X}\right)^{2}}{8-1}} ; \\
& D_{Y}(\tilde{\tilde{A}}, \tilde{\tilde{B}})=\sqrt{\frac{\sum_{i=1}^{8}\left(y_{i}-\bar{Y}\right)^{2}}{8-1}} .
\end{aligned}
$$

\section{CAM - client acceptance method for audit firms}

In this section, we use the TOPSIS approach and Chen and Sanguansat's (2011) similarity measure method to solve a fuzzy analysis problem. Specifically, we examine the client acceptance method (CAM) by audit firms. The flowchart of using CAM is shown in Figure 2.

\subsection{Decision environment for client acceptance in an audit firm}

Assume that there are $\mathrm{n}$ potential new clients $C_{1}, C_{2}, C_{3}, \ldots, C_{n}$ for an audit firm. A decision maker (DM) in the audit firm should consider $\mathrm{k}$ attributes $A_{1}, A_{2}, A_{3}, \ldots, A_{k}$ in the decision-making process when accepting new clients. According to the subjective decision makers' opinions, we use a nine-member linguistic term set, shown in Table 1, to represent the linguistic terms and their corresponding IVFNs. These IVFNs determine each attribute's weighting and, therefore, the attribute score for each new client in the evaluation process. 


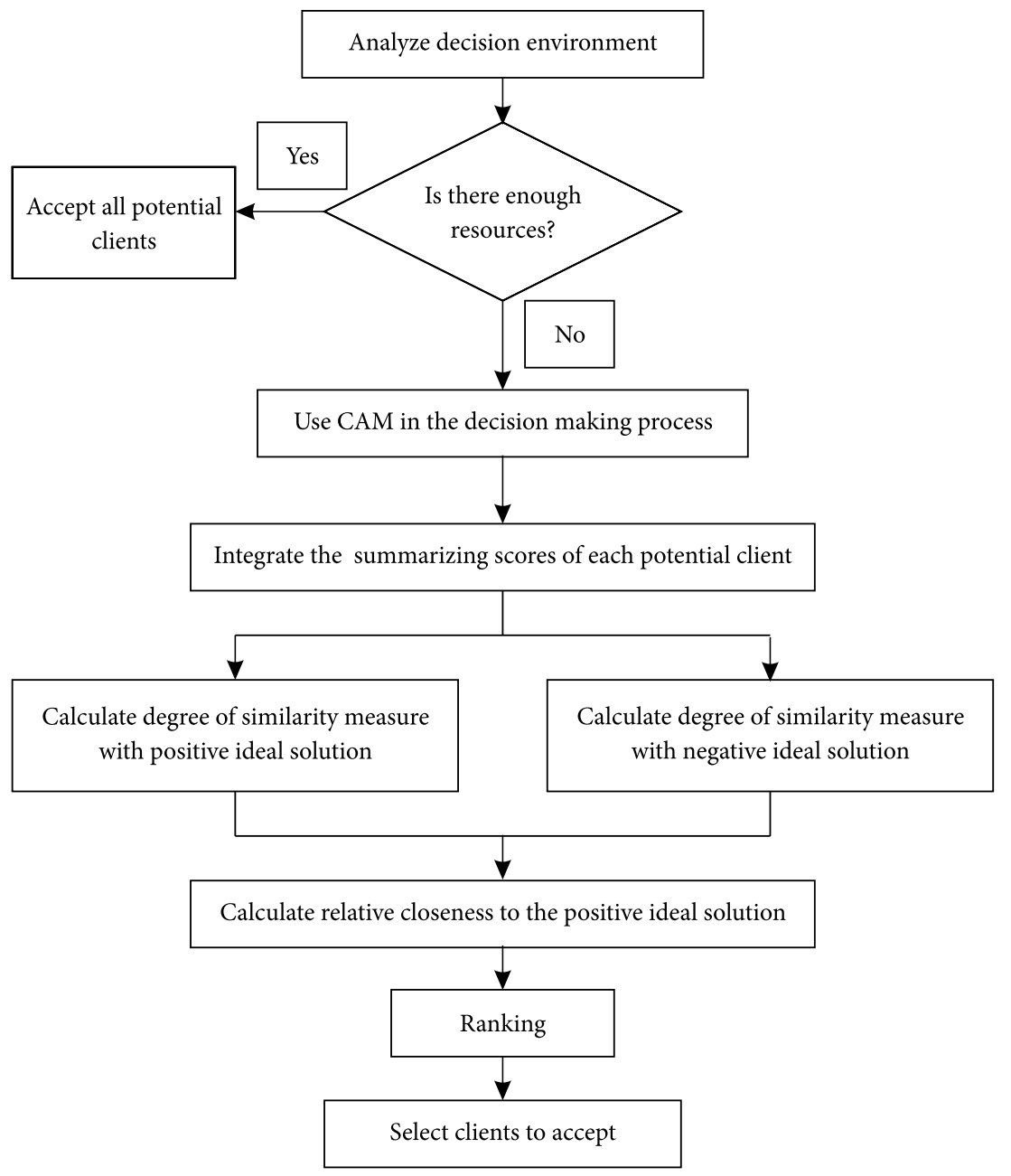

Fig. 2. Flowchart of using CAM

Table 1. Nine-member linguistic terms and their corresponding interval-valued fuzzy numbers

\begin{tabular}{ll}
\hline \multicolumn{1}{c}{ Linguistic terms } & \multicolumn{1}{c}{ Interval-valued trapezoidal fuzzy numbers } \\
\hline Absolutely low & {$[(0.0,0.0,0.0,0.0 ; 1.0),(0.0,0.0,0.0,0.0 ; 1.0)]$} \\
Very low & {$[(0.0075,0.0075,0.015,0.0525 ; 0.5),(0.0,0.0,0.02,0.07 ; 1.0)]$} \\
Low & {$[(0.0875,0.12,0.16,0.1825 ; 0.5),(0.04,0.10,0.18,0.23 ; 1.0)]$} \\
Fairly low & {$[(0.2325,0.255,0.325,0.3575 ; 0.5),(0.17,0.22,0.36,0.42 ; 1.0)]$} \\
Medium & {$[(0.4025,0.4525,0.5375,0.5675 ; 0.5),(0.32,0.41,0.58,0.65 ; 1.0)]$} \\
Fairly high & {$[(0.65,0.6725,0.7575,0.79 ; 0.5),(0.58,0.63,0.80,0.86 ; 1.0)]$} \\
High & {$[(0.7825,0.815,0.885,0.9075 ; 0.5),(0.72,0.78,0.92,0.97 ; 1.0)]$} \\
Very high & {$[(0.9475,0.985,0.9925,0.9925 ; 0.5),(0.93,0.98,1.0,1.0 ; 1.0)]$} \\
Absolutely high & {$[(1.0,1.0,1.0,1.0 ; 1.0),(1.0,1.0,1.0,1.0 ; 1.0)]$} \\
\hline
\end{tabular}

Source: Chen, S. J. and Chen, S. M. (2009). 
Here, we briefly describe notation used in the proposed method. $C_{i}$ denotes the $i^{\text {th }}$ potential new client being considered; $A_{j}$ denotes the $j^{\text {th }}$ attribute considered; and $c_{i j}$ denotes the score in linguistic terms of the $i^{\text {th }}$ potential new client with respect to the $j^{\text {th }}$ attribute. The decision matrix is shown below:

$$
\begin{aligned}
& \begin{array}{llllll}
A_{1} & A_{2} & \cdots & A_{j} & \cdots & A_{k}
\end{array} \\
& \mathrm{D}=\frac{C_{1}}{C_{2}} \begin{array}{c}
C_{21} \\
C_{i} \\
C_{n}
\end{array}\left[\begin{array}{ccccc}
c_{11} & c_{12} & \cdots & c_{1 j} \cdots & c_{1 k} \\
c_{21} & c_{22} & \cdots & c_{2 j} \cdots & c_{2 k} \\
\vdots & \vdots & & \vdots & \vdots \\
c_{i 1} & c_{i 2} & \cdots & c_{i j} \cdots & c_{i k} \\
c_{n 1} & c_{n 2} & \cdots & c_{n j} \cdots & c_{n k}
\end{array}\right] .
\end{aligned}
$$

In the proposed method, we used the linguistic terms for the weight $\varpi_{j}$ (importance) of each attribute $A_{j}$ that could be denoted in an IVFN $\tilde{\tilde{\sigma}}_{j}$. For example, if decision makers considered the level of importance for their client's audit fee (first attribute) to be "very high", the IVFN of the first attribute's weighting could be represented as $\tilde{\tilde{\tilde{\omega}}}_{1}(=[(0.9475,0.985,0.9925,0.9925 ; 0.5),(0.93,0.98,1.0,1.0 ; 1.0)])$. We attempt to normalize the various weights, which allows for comparison across the weights. Therefore, $\tilde{\tilde{\omega}}_{j}$ denotes the normalized linguistic IVFN for the importance of each attribute. After integrating, the IVFN of weighted scores for potential client $C_{i}$ in attribute $A_{j}$ is denoted as $\tilde{\tilde{C}}_{i j}$. Simultaneously, $\tilde{\tilde{C}}_{i j}^{\star}$ and $\tilde{\tilde{C}}_{i j}$ denote the linguistic IVFN for the weighted positive ideal solution and the linguistic IVFN for the weighted negative ideal solution, respectively.

The proposed algorithm for client acceptance decision-making is presented in the following section.

\subsection{Integrating the summarizing scores for potential clients using IVFNs}

In the client-acceptance evaluation process, a decision maker considers several attributes. Each attribute might have its own weight in the process. We normalize the IVFN of weight $\left.\tilde{\tilde{\tilde{\varpi}}}_{j}\left(=\left(\varpi_{j 1}^{\mathrm{L}}, \varpi_{j 2}^{\mathrm{L}}, \varpi_{j 3}^{\mathrm{L}}, \varpi_{j 4}^{\mathrm{L}} ; w_{\tilde{\tilde{\tilde{\omega}}}_{j}}^{\mathrm{L}}\right),\left(\varpi_{j 1}^{\mathrm{U}}, \varpi_{j 2}^{\mathrm{U}}, \varpi_{j 3}^{\mathrm{U}}, \varpi_{j 4}^{\mathrm{U}} ; w_{\tilde{\tilde{\tilde{\omega}}}_{j}}^{\mathrm{U}}\right)\right]\right)$ as $\tilde{\tilde{\omega}}_{j}$ with the following arithmetic operation:

$$
\begin{gathered}
\tilde{\tilde{\omega}}_{j}=\frac{\left[\left(\varpi_{j 1}^{\mathrm{L}}, \varpi_{j 2}^{\mathrm{L}}, \varpi_{j 3}^{\mathrm{L}}, \varpi_{j 4}^{\mathrm{L}} ; w_{\tilde{\tilde{\tilde{\omega}}}_{j}}^{\mathrm{L}}\right),\left(\varpi_{j 1}^{\mathrm{U}}, \varpi_{j 2}^{\mathrm{U}}, \varpi_{j 3}^{\mathrm{U}}, \varpi_{j 4}^{\mathrm{U}} ; w_{\tilde{\tilde{\omega}}_{j}}^{\mathrm{U}}\right)\right]}{\sum_{j=1}^{k}\left[\left(\varpi_{j 1}^{\mathrm{L}}, \varpi_{j 2}^{\mathrm{L}}, \varpi_{j 3}^{\mathrm{L}}, \varpi_{j 4}^{\mathrm{L}} ; w_{\tilde{\tilde{\tilde{\omega}}}_{j}}^{\mathrm{L}}\right),\left(\varpi_{j 1}^{\mathrm{U}}, \varpi_{j 2}^{\mathrm{U}}, \varpi_{j 3}^{\mathrm{U}}, \varpi_{j 4}^{\mathrm{U}} ; w_{\tilde{\tilde{\tilde{\omega}}}_{j}}^{\mathrm{U}}\right)\right]}= \\
{\left[\left(\omega_{j 1}^{\mathrm{L}}, \omega_{j 2}^{\mathrm{L}}, \omega_{j 3}^{\mathrm{L}}, \omega_{j 4}^{\mathrm{L}} ; w_{\tilde{\tilde{\omega}}_{j}}^{\mathrm{L}}\right),\left(\omega_{j 1}^{\mathrm{U}}, \omega_{j 2}^{\mathrm{U}}, \omega_{j 3}^{\mathrm{U}}, \omega_{j 4}^{\mathrm{U}} ; w_{\tilde{\tilde{\omega}}_{j}}^{\mathrm{U}}\right)\right] .}
\end{gathered}
$$

For example, after reviewing the information for a new potential client $C_{1}$, the decision maker may consider that the scores for the audit fee (ex. $\left.A_{1}\right)$ and business model (ex. $A_{2}$ ) are "low" and "fairly high", respectively. To calculate the IVFN of weighted scores of potential client $C_{i}$ in the attribute $A_{j}, \widetilde{\tilde{C}}_{i j}$, we should integrate the scores, $\tilde{\tilde{c}}_{i j}$, under its weighting. We use the following equation: 


$$
\begin{aligned}
\tilde{\tilde{C}}_{i j}= & \tilde{\tilde{c}}_{i j} \otimes \tilde{\tilde{\omega}}_{j}= \\
& {\left[\left(c_{i j 1}^{\mathrm{L}}, c_{i j 2}^{\mathrm{L}}, c_{i j 3}^{\mathrm{L}}, c_{i j 4}^{\mathrm{L}} ; w_{\tilde{\tilde{c}}_{i j}}^{\mathrm{L}}\right),\left(c_{i j 1}^{\mathrm{U}}, c_{i j 2}^{\mathrm{U}}, c_{i j 3}^{\mathrm{U}}, c_{i j 4}^{\mathrm{U}} ; w_{\tilde{\tilde{c}}_{i j}}^{\mathrm{U}}\right)\right] \otimes } \\
& {\left[\left(\omega_{j 1}^{\mathrm{L}}, \omega_{j 2}^{\mathrm{L}}, \omega_{j 3}^{\mathrm{L}}, \omega_{j 4}^{\mathrm{L}} ; w_{\tilde{\tilde{\omega}}_{j}}^{\mathrm{L}}\right),\left(\omega_{j 1}^{\mathrm{U}}, \omega_{j 2}^{\mathrm{U}}, \omega_{j 3}^{\mathrm{U}}, \omega_{j 4}^{\mathrm{U}} ; w_{\tilde{\tilde{\omega}}_{j}}^{\mathrm{U}}\right)\right]=} \\
& {\left[\left(C_{i j 1}^{\mathrm{L}}, C_{i j 2}^{\mathrm{L}}, C_{i j 3}^{\mathrm{L}}, C_{i j 4}^{\mathrm{L}} ; w_{\tilde{\tilde{C}}_{i j}}^{\mathrm{L}}\right),\left(C_{i j 1}^{\mathrm{U}}, C_{i j 2}^{\mathrm{U}}, C_{i j 3}^{\mathrm{U}}, C_{i j 4}^{\mathrm{U}} ; w_{\tilde{\tilde{C}}_{i j}}^{\mathrm{U}}\right)\right] . }
\end{aligned}
$$

Because of the difficulties in ranking of IVFNs directly, we used the TOPSIS method after calculating the IVFN of weighted scores for potential client $\tilde{\tilde{C}}_{i}$ in each attribute. Following the TOPSIS method, we used the fixed ideal solution to simplify the calculations of the similarity measure between the IVFNs. The similarity measures with a positive ideal solution and a negative ideal solution are calculated in Sections 3.3 and 3.4, respectively.

\subsection{Similarity measure with positive ideal solution}

To simplify the calculations, we rank the potential new clients according to the decision-making attributes and give the score of the ideal solution for "excellence", which was denoted by theIVFN as $\left[\left(c_{i j 1}^{*}, c_{i j 2}^{*}, c_{i j 3}^{*}, c_{i j 4}^{*} ; w_{\tilde{C}_{i j}^{*}}^{\mathrm{L}}\right),\left(c_{i j 1}^{*}, c_{i j 2}^{*}, c_{i j 3}^{*}, c_{i j 4}^{*} ; w_{\tilde{\tilde{C}}_{i j}^{*}}^{\mathrm{U}}\right)\right]$. The IVFN for the weighted ideal solution, $C_{i j}^{*}$, is shown as follows:

$$
\begin{aligned}
\tilde{\tilde{C}}_{i j}^{*}= & {\left[\left(c_{i j 1}^{\star \mathrm{L}}, c_{i j 2}^{* \mathrm{~L}}, c_{i j 3}^{\star} \mathrm{L}, c_{i j 4}^{\star \mathrm{L}} ; w_{\tilde{\tilde{C}}_{i j}^{*}}^{\mathrm{L}}\right),\left(c_{i j 1}^{*}, c_{i j 2}^{*}, c_{i j 3}^{\star \mathrm{U}}, c_{i j 4}^{*} ; w_{\tilde{\tilde{C}}_{i j}^{*}}^{\mathrm{U}}\right)\right] \otimes } \\
& {\left[\left(\omega_{i j 1}^{\mathrm{L}}, \omega_{i j 2}^{\mathrm{L}}, \omega_{i j 3}^{\mathrm{L}}, \omega_{i j 4}^{\mathrm{L}} ; w_{\tilde{\tilde{\omega}}_{i j}}^{\mathrm{L}}\right),\left(\omega_{i j 1}^{\mathrm{U}}, \omega_{i j 2}^{\mathrm{U}}, \omega_{i j 3}^{\mathrm{U}}, \omega_{i j 4}^{\mathrm{U}} ; w_{\tilde{\tilde{\omega}}_{i j}}^{\mathrm{U}}\right)\right]=} \\
& {[(1.0,1.0,1.0,1.0 ; 1.0),(1.0,1.0,1.0,1.0 ; 1.0)] \otimes } \\
& {\left[\left(\omega_{i j 1}^{\mathrm{L}}, \omega_{i j 2}^{\mathrm{L}}, \omega_{i j 3}^{\mathrm{L}}, \omega_{i j 4}^{\mathrm{L}} ; w_{\tilde{\tilde{\omega}}_{i j}}^{\mathrm{L}}\right),\left(\omega_{i j 1}^{\mathrm{U}}, \omega_{i j 2}^{\mathrm{U}}, \omega_{i j 3}^{\mathrm{U}}, \omega_{i j 4}^{\mathrm{U}} ; w_{\tilde{\tilde{\omega}}_{i j}}^{\mathrm{U}}\right)\right]=} \\
& {\left[\left(\omega_{i j 1}^{\mathrm{L}}, \omega_{i j 2}^{\mathrm{L}}, \omega_{i j 3}^{\mathrm{L}}, \omega_{i j 4}^{\mathrm{L}} ; w_{\tilde{\tilde{\omega}}_{i j}}^{\mathrm{L}}\right),\left(\omega_{i j 1}^{\mathrm{U}}, \omega_{i j 2}^{\mathrm{U}}, \omega_{i j 3}^{\mathrm{U}}, \omega_{i j 4}^{\mathrm{U}} ; w_{\tilde{\tilde{\omega}}_{i j}}^{\mathrm{U}}\right)\right] . }
\end{aligned}
$$

We use the proposed similarity measure to evaluate the degree of similarity $S\left(\tilde{\tilde{C}}_{i j}, \tilde{\tilde{C}}_{i j}^{*}\right)$ between the IVFNs of the weighted score for potential client $\tilde{\tilde{C}}_{i j}$ and the weighted ideal solution $\tilde{\tilde{C}}_{i j}^{\star}$ as follows:

$$
S\left(\tilde{\tilde{C}}_{i j}, \tilde{\tilde{C}}_{i j}^{*}\right)=D C\left(\tilde{\tilde{C}}_{i j}^{U}, \tilde{\tilde{C}}_{i j}^{\star U}\right) \times\left(1-D D_{X}\left(\tilde{\tilde{C}}_{i j}, \tilde{\tilde{C}}_{i j}^{*}\right)\right) \times\left(1-D D_{Y}\left(\tilde{\tilde{C}}_{i j}, \tilde{\tilde{C}}_{i j}^{*}\right)\right) .
$$

As mentioned in Section 2, the degree of similarity is affected by the degree of closeness between the upper trapezoidal fuzzy numbers of the weighted scores for a potential client for attribute $A_{j}$ and the weighted ideal solution on the X-axis, $D C\left(\tilde{\tilde{C}}_{i j}^{\mathrm{U}}, \tilde{\tilde{C}}_{i j}^{*} \mathrm{U}\right)$ :

$$
\operatorname{DC}\left(\tilde{\tilde{C}}_{i j}^{\mathrm{U}}, \tilde{\tilde{C}}_{i j}^{* \mathrm{U}}\right)=1-\frac{\sum_{k=1}^{4}\left(c_{i j k}^{\mathrm{U}}-\omega_{i j k}^{\mathrm{U}}\right)}{4} .
$$


In addition, the degrees of difference between the shapes of the IVFNs for the weighted scores for a potential client and weighted ideal solution on the $\mathrm{X}$-axis and Y-axis, $D D_{X}\left(\tilde{\tilde{C}}_{i j}, \tilde{\tilde{C}}_{i j}^{*}\right)$ and $D D_{Y}\left(\tilde{\tilde{C}}_{i j}, \tilde{\tilde{C}}_{i j}^{*}\right)$ could also affect the degree of similarity:

$$
\begin{aligned}
& D D_{X}\left(\tilde{\tilde{C}}_{i j}, \tilde{\tilde{C}}_{i j}^{*}\right)=\sqrt{\frac{\sum_{l=1}^{8}\left(x_{l}^{*}-\bar{X}^{*}\right)^{2}}{8-1}} ; \\
& D D_{Y}\left(\tilde{\tilde{C}}_{i j}, \tilde{\tilde{C}}_{i j}^{*}\right)=\sqrt{\frac{\sum_{l=1}^{8}\left(y_{l}^{*}-\bar{Y}^{*}\right)^{2}}{8-1}} .
\end{aligned}
$$

Let $P^{*}$ denote the difference between the shapes of the IVFNs for the weighted scores for a potential client and the weighted ideal solution on the $\mathrm{X}$-axis, and $\bar{X}^{\star}$ denotes the average of $P^{*}$ :

$$
\begin{aligned}
P^{*}= & {\left[x_{1}^{*}, x_{2}^{*}, x_{3}^{*}, x_{4}^{*}, x_{5}^{*}, x_{6}^{*}, x_{7}^{*}, x_{8}^{*}\right]=} \\
& {\left[c_{i j 1}^{* \mathrm{~L}}-1, c_{i j 2}^{* \mathrm{~L} 2}-1, c_{i j 3}^{* \mathrm{~L}}-1, c_{i j 4}^{* \mathrm{~L}}-1, c_{i j 1}^{* \mathrm{U}}-1, c_{i j 2}^{* \mathrm{U}}-1, c_{i j 3}^{* \mathrm{U}}-1, c_{i j 4}^{* \mathrm{U}}-1\right] ; } \\
\bar{X}^{*}= & \frac{\sum_{k=1}^{4} c_{i j k}^{*}+\sum_{k=1}^{4} c_{i j k}^{*}}{8}-1 .
\end{aligned}
$$

Let $Q^{*}$ denote the difference between the shapes of the IVFNs for the weighted scores for a potential client and the weighted ideal solution on the Y-axis, and $\bar{Y}^{*}$ denotes the average of $Q^{*}$ :

$$
\begin{aligned}
Q^{*}= & {\left[y_{1}^{*}, y_{2}^{*}, y_{3}^{*}, y_{4}^{*}, y_{5}^{*}, y_{6}^{*}, y_{7}^{*}, y_{8}^{*}\right]=} \\
& {\left[0, w_{\tilde{\tilde{C}}_{i j}}^{\mathrm{L}}-w_{\tilde{\tilde{\omega}}_{i j}}^{\mathrm{L}}, w_{\tilde{\tilde{C}}_{i j}}^{\mathrm{L}}-w_{\tilde{\tilde{\omega}}_{i j}}^{\mathrm{L}}, 0,0, w_{\tilde{\tilde{C}}_{i j}}^{\mathrm{U}}-w_{\tilde{\tilde{\omega}}_{i j}}^{\mathrm{U}}, w_{\tilde{\tilde{C}}_{i j}}^{\mathrm{U}}-w_{\tilde{\tilde{\omega}}_{i j}}^{\mathrm{U}}, 0\right] ; } \\
\bar{Y}^{*}= & \frac{w_{\tilde{\tilde{C}}_{i j}}^{\mathrm{L}}+w_{\tilde{\tilde{C}}_{i j}}^{\mathrm{U}}-w_{\tilde{\tilde{\omega}}_{i j}}^{\mathrm{L}}-w_{\tilde{\tilde{\omega}}_{i j}}^{\mathrm{U}}}{4} .
\end{aligned}
$$

\subsection{Similarity measure with a negative ideal solution}

As mentioned in above, to rank a potential new client according to the decision-making attributes, we take the score of the negative ideal solution as "harmful", which was denoted in the IVFN as $\left[\left(c_{i j 1}^{\mathrm{L} 1}, c_{i j 2}^{\mathrm{L}}, c_{i j 3}^{\mathrm{L}}, c_{i j 4}^{\mathrm{L}} ; w_{\tilde{\tilde{C}}_{\overline{i j}}^{\mathrm{L}}}^{\mathrm{L}}\right),\left(c_{i j 1}^{\mathrm{U}}, c_{i j 2}^{\mathrm{U}}, c_{i j 3}^{\mathrm{U}}, c_{i j 4}^{\mathrm{U}} ; w_{\tilde{\tilde{C}}_{\overline{i j}}}^{\mathrm{U}}\right)\right]$. The IVFN for the weighted negative ideal solution, $\tilde{\tilde{C}}_{\overline{i j}}$, is shown as follows:

$$
\begin{gathered}
\tilde{\tilde{C}}_{\overline{i j}}=\left[\left(c_{i j 1}^{\mathrm{L}}, c_{i j 2}^{\mathrm{L}}, c_{i j 3}^{-\mathrm{L}}, c_{i j 4}^{\mathrm{L}} ; w_{\tilde{\tilde{C}}_{i \bar{j}}^{\mathrm{L}}}^{\mathrm{L}}\right),\left(c_{i j 1}^{\mathrm{U}}, c_{\overline{i j 2} 2}^{\mathrm{U}}, c_{i \overline{-i j 3}}^{\mathrm{U}}, c_{i j 4}^{\mathrm{U}} ; w_{\tilde{\tilde{C}}_{\overline{i j}}}^{\mathrm{U}}\right)\right] \otimes \\
{\left[\left(\omega_{i j 1}^{\mathrm{L}}, \omega_{i j 2}^{\mathrm{L}}, \omega_{i j 3}^{\mathrm{L}}, \omega_{i j 4}^{\mathrm{L}} ; w_{\tilde{\tilde{\omega}}_{i j}}^{\mathrm{L}}\right),\left(\omega_{i j 1}^{\mathrm{U}}, \omega_{i j 2}^{\mathrm{U}}, \omega_{i j 3}^{\mathrm{U}}, \omega_{i j 4}^{\mathrm{U}} ; w_{\tilde{\tilde{\omega}}_{i j}}^{\mathrm{U}}\right)\right]=}
\end{gathered}
$$


$[(0,0,0,0 ; 1.0),(0,0,0,0 ; 1.0)] \otimes\left[\left(\omega_{i j 1}^{\mathrm{L}}, \omega_{i j 2}^{\mathrm{L}}, \omega_{i j 3}^{\mathrm{L}}, \omega_{i j 4}^{\mathrm{L}} ; w_{\tilde{\tilde{\omega}}_{i j}}^{\mathrm{L}}\right),\left(\omega_{i j 1}^{\mathrm{U}}, \omega_{i j 2}^{\mathrm{U}}, \omega_{i j 3}^{\mathrm{U}}, \omega_{i j 4}^{\mathrm{U}} ; w_{\tilde{\tilde{\omega}}_{i j}}^{\mathrm{U}}\right)\right]=$ $\left[\left(0,0,0,0 ; w_{\tilde{\tilde{\omega}}_{i j}}^{\mathrm{L}}\right),\left(0,0,0,0 ; w_{\tilde{\tilde{\omega}}_{i j}}^{\mathrm{U}}\right)\right]$.

We use the proposed similarity measure to evaluate the degree of similarity $S\left(\tilde{\tilde{C}}_{i j}, \tilde{\tilde{C}}_{i \overline{\tilde{C}}}\right)$ between the IVFNs of $\tilde{\tilde{C}}_{i j}$ and the negative ideal solution, $\tilde{\tilde{C}}_{i \bar{j}}$ :

$$
S\left(\tilde{\tilde{C}}_{i j}, \tilde{\tilde{C}}_{i \bar{j}}\right)=D C\left(\tilde{\tilde{C}}_{i j}^{U}, \tilde{\tilde{C}}_{i \bar{j}}^{\mathrm{U}}\right) \times\left(1-D D_{X}\left(\tilde{\tilde{C}}_{i j}, \tilde{\tilde{C}}_{\overline{i j}}\right)\right) \times\left(1-D D_{Y}\left(\tilde{\tilde{C}}_{i j}, \tilde{\tilde{C}}_{i \bar{j}}\right)\right) .
$$

As mentioned in Section 2, the degree of similarity is affected by the degree of closeness between the upper trapezoidal fuzzy numbers of the weighted scores of potential clients for attribute $A_{j}$, and the weighted negative ideal solution on the $\mathrm{X}$-axis, $D C\left(\tilde{\tilde{C}}_{i j}^{\mathrm{U}}, \tilde{\tilde{C}}_{i \bar{j}}^{\mathrm{U}}\right)$ :

$$
D C\left(\tilde{\tilde{C}}_{i j}^{\mathrm{U}}, \tilde{\tilde{C}}_{i j}^{\mathrm{U}}\right)=1-\frac{\sum_{k=1}^{4} c_{i j k}^{\mathrm{U}}}{4} .
$$

In addition, the degrees of difference between the shapes of the IVFNs for the scores of potential clients and the negative ideal solution on the X-axis and Y-axis, $D D_{X}\left(\tilde{\tilde{C}}_{i j}, \tilde{\tilde{C}}_{i j}\right)$ and $D D_{Y}\left(\tilde{\tilde{C}}_{i j}, \tilde{\tilde{C}}_{i \bar{j}}\right)$, respectively, could also affect the degree of similarity:

$$
\begin{gathered}
D D_{X}\left(\tilde{\tilde{C}}_{i j}, \tilde{\tilde{C}}_{i j}\right)=\sqrt{\frac{\sum_{l=1}^{8}\left(x_{\bar{l}}-\bar{X}-\right)^{2}}{8-1}} ; \\
D D_{Y}\left(\tilde{\tilde{C}}_{i j}, \tilde{\tilde{C}}_{i j}\right)=\sqrt{\frac{\sum_{l=1}^{8}\left(y_{\bar{l}}-\bar{Y}-\right)^{2}}{8-1}} .
\end{gathered}
$$

Let $P$ - denote the difference between the shapes of the IVFNs for the weighted scores of a potential client and a weighted negative ideal solution on the $\mathrm{X}$-axis and $\bar{X}$ - denote the average of $P_{-}$:

$$
\begin{aligned}
& P_{-}=\left[x_{1}^{-}, x_{2}^{-}, x_{3}^{-}, x_{4}^{-}, x_{5}^{-}, x_{6}^{-}, x_{7}^{-}, x_{8}^{-}\right]= \\
& {\left[c_{i j 1} \mathrm{~L}, c_{i j 2} \mathrm{~L}, c_{i j 3}^{-}, c_{i j 4}^{-}, c_{i j 1}^{-} \mathrm{U}, c_{i j 2}^{-\mathrm{U}}, c_{i j 3}^{-\mathrm{U}}, c_{i j 4}^{-\mathrm{U}}\right] \text {; }} \\
& \bar{X}_{-}=\frac{\sum_{k=1}^{4} c_{i j k}^{\mathrm{L}}+\sum_{k=1}^{4} c_{i j k}^{\mathrm{U}}}{8} .
\end{aligned}
$$

Let $Q$ - denote the difference between the shapes of the IVFNs for the scores of a potential client and negative ideal solution on the $\mathrm{Y}$-axis and $\bar{Y}-$ denote the average of $Q^{-}$:

$$
\begin{aligned}
Q^{-}= & {\left[y_{1}^{-}, y_{2}^{-}, y_{3}^{-}, y_{4}^{-}, y_{5}^{-}, y_{6}^{-}, y_{7}^{-}, y_{8}^{-}\right]=} \\
& {\left[0, w_{\tilde{\tilde{C}}_{i j}}^{\mathrm{L}}-w_{\tilde{\tilde{\omega}}_{i j}}^{\mathrm{L}}, w_{\tilde{\tilde{C}}_{i j}}^{\mathrm{L}}-w_{\tilde{\tilde{\omega}}_{i j}}^{\mathrm{L}}, 0,0, w_{\tilde{\tilde{C}}_{i j}}^{\mathrm{U}}-w_{\tilde{\tilde{\omega}}_{i j}}^{\mathrm{U}}, w_{\tilde{\tilde{C}}_{i j}}^{\mathrm{U}}-w_{\tilde{\tilde{\omega}}_{i j}}^{\mathrm{U}}, 0\right] ; }
\end{aligned}
$$




$$
\bar{Y}-=\frac{w_{\tilde{\tilde{C}}_{i j}}^{\mathrm{L}}+w_{\tilde{\tilde{C}}_{i j}}^{\mathrm{U}}-w_{\tilde{\tilde{\omega}}_{i j}}^{\mathrm{L}}-w_{\tilde{\tilde{\omega}}_{i j}}^{\mathrm{U}}}{4}
$$

\subsection{Relative closeness to the positive ideal solution}

Finally, we calculate $R C_{i^{\star}}$, the relative closeness to the positive ideal solution. The relative closeness $R C_{i^{\star}}$ of the IVFN score for potential client $\tilde{\tilde{C}}_{i}$ with respect to positive ideal solution $\tilde{\widetilde{C}}^{\star}$ is defined as follows:

$$
R C_{i^{\star}}=\frac{\left(\sum_{j=1}^{k} S\left(\tilde{\tilde{C}}_{i j}, \tilde{\tilde{C}}_{i j}^{\star}\right)\right) / k}{\left(\sum_{j=1}^{k} S\left(\tilde{\tilde{C}}_{i j}, \tilde{\tilde{C}}_{i j}^{\star}\right)\right) / k+\left(\sum_{j=1}^{k} S\left(\tilde{\tilde{C}}_{i j}, \tilde{\tilde{C}}_{i j}\right)\right) / k}, \quad 0<R C_{i^{\star}}<1 .
$$

The largest average degree of similarity between a potential client and the positive ideal solution $\left(\left(\sum_{j=1}^{k} S\left(\tilde{\tilde{C}}_{i j}, \tilde{\tilde{C}}_{i j}^{\star}\right)\right) / k\right)$ and the smallest average degree of similarity between a potential client and the negative ideal solution $\left(\left(\sum_{j=1}^{k} S\left(\tilde{\tilde{C}}_{i j}, \tilde{\tilde{C}}_{i j}\right)\right) / k\right)$ will result in the largest relative closeness, $R C_{i *}$. Finally, a set of potential new clients can be ranked according to preference in descending order of $R C_{i *}$.

\subsection{Algorithm}

The proposed fuzzy decision-making algorithm steps are as follows:

Step 1. Modify the linguistic values $\tilde{\tilde{\omega}}_{j}$ of the importance of each attribute $A_{j}$ to $\tilde{\tilde{\omega}}_{j}$ (Eq. (9)).

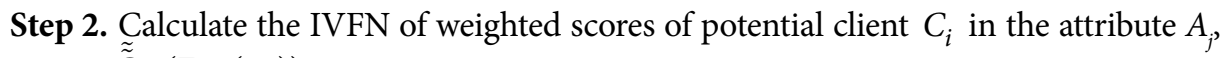
$\tilde{\tilde{C}}_{i j}$ (Eq. (10)).

Step 3. Use the proposed similarity measure to evaluate the degrees of similarity between the IVFNs of $\tilde{\tilde{C}}_{i j}$ and the outcome of the weighted positive ideal solution $\tilde{\tilde{C}}_{i j}^{*}$ (Eqs (11)-(19)).

Step 4. Use the proposed similarity measure to evaluate the degrees of similarity between the IVFNs of $\widetilde{\tilde{C}}_{i j}$ and the outcome of the weighted negative ideal solution $\widetilde{\tilde{C}}_{i j}$ (Eqs (20)-(28)).

Step 5. Calculate the relative closeness $R C_{i^{\star}}$ to the positive ideal solution (Eq. (29)).

Step 6. Rank the preference order.

\section{A case study of client-acceptance decision making}

In this section, we use a case study to apply the proposed method to one of the four largest international audit firms (the Big 4) in Taiwan. Through interviews with some audit partners in the audit firms, we summarize the factors that are difficult to evaluate in the client acceptance 
decision-making process. Based on these factors, we examine the proposed acceptance method of three audit partners whose profile in this aspect of auditing is briefly listed as follows:

- DM1 has seventeen years of work experience in a "Big 4" audit firm in Taiwan. DM1's clients are primarily in the consumer electronics and medical supplies industries. DM1 has engagements with at least three listed corporations. Additionally, DM1 is also involved with many unlisted companies that mostly belong to the manufacturing industry.

- DM2 has twenty-three years of work experience in the same audit firm as DM1. DM2's clients are primarily in the fiber optic transmission components and electronic components industry. DM2 has engagements with at least three listed corporations. Otherwise, most of DM2's clients are foreign companies.

- DM3 also has twenty-three years of work experience in the same audit firm as DM1 and DM2. DM3's clients are primarily in the consumer electronics industry. DM3 has engagements with at least two listed corporations in the audit firm. One of the clients is the headquarters of a multi-national corporation whose products have successfully gained a significant market share in the global sector. Additionally, DM3 is also involved in many unlisted companies that mostly belong to the manufacturing industry.

First, we asked these three audit partners to determine the weight for each factor. Then, we showed them five situations for potential new clients and asked for their opinions of each factor of client-acceptance decisions. Simultaneously, we asked them to rank these five potential new clients for acceptance. Finally, we compared the ranking they constructed with the one we calculated via the proposed method.

\subsection{In-depth interview}

For the case study, we interviewed each of the three audit partners introduced above. Through the interviews, we learned that, at the audit firm, they apply different client acceptance processes according to the potential clients' circumstances. If the potential client is a listed company, they will form a committee to manage the risk to the audit firm. In the committee, the client's acceptance will be approved by a vote. If the potential client is not a listed company, however, the audit engagement partner evaluates the risks and makes the client acceptance decision directly. According to the interviews, there are four major factors that they consider to be characterized by uncertainty and complexity. These factors are benefit, enterprise image, industrial prospects and financial status. Based on the opinions of the interviewees, the four factors are briefly described as follows:

- "Benefit" mainly refers to the audit fee from a potential client. However, the audit fee is not the only factor decision makers use to evaluate the potential benefit of taking on a client. They also consider other potential clients that the new client will bring to the audit firm. For example, if the new client is affiliated with other corporations, those affiliated corporations may become the audit firm's clients in the future. Therefore, the benefit the decision makers consider will also include the potential benefit of these affiliates in the near future.

- "Enterprise image" refers to the presence of the corporation in newspapers or other mass media. The term includes both news events and advertisements carried by any 
mass media outlet. In addition, the mass media appearances of the corporation's directors and management of is considered to be a part of enterprise image.

- "Industrial prospects" is the firm's business forecast and valuation. It includes competitiveness in the industry, business strategy analysis, accounting analysis, and financial analysis. Therefore, decision makers may consider attributes of the company's industrial category and business model, as well as other considerations.

- "Financial status" refers to the company's operating results and its financial structure. Simultaneously, whether the company will be a going concern is taken into consideration in the decision-making process.

We inquired into the weight that each factor was given based on the opinions of the three audit partners using nine-member linguistic terms as shown in Table 2.

Table 2. Linguistic values of four attributes' weighting according to decision makers

\begin{tabular}{cllll}
\hline $\begin{array}{c}\text { Decision } \\
\text { Maker }\end{array}$ & \multicolumn{1}{c}{$\begin{array}{c}\varpi_{1}: \\
\text { Benefit }\end{array}$} & Enterprise image & Industrial prospect & Financial status \\
\hline DM \#1 & Absolutely high & Medium & Fairly high & Fairly high \\
DM \#2 & Absolutely high & High & Fairly low & Fairly high \\
DM \#3 & Very high & Absolutely high & Medium & Very high \\
\hline
\end{tabular}

After summarizing the weight given to the four factors by the three audit partners, we selected five circumstances for potential new clients and asked the three audit partners' to score each factor using the nine-member linguistic terms mentioned above. Considering the convenience of performing the calculations according to the proposed method, we expected that because these five potential clients are not listed companies, the acceptance decision will be made by one decision maker. We briefly describe these five potential clients' circumstances in Table 3.

The scores for the five situations from each decision maker are shown in Table 4 through Table 6.

\subsection{Using CAM in the case study}

In the following, we use the proposed algorithm to solve the client acceptance problem using DM1's opinion.

Step 1. Based on Eq. (9), we modify the importance $\tilde{\tilde{\tilde{\omega}}}_{j}$ of each attributes $A_{j}$ by DM1 to $\tilde{\tilde{\omega}}_{j}$ :

$$
\begin{aligned}
D M_{1}: & \\
\tilde{\tilde{\omega}}_{1}= & \tilde{\tilde{\tilde{\omega}}}_{1} \varnothing\left(\tilde{\tilde{\tilde{\omega}}}_{1} \oplus \tilde{\tilde{\tilde{\omega}}}_{2} \oplus \tilde{\tilde{\tilde{\omega}}}_{3} \oplus \tilde{\tilde{\tilde{\omega}}}_{4}\right)=[(1.0,1.0,1.0,1.0 ; 1.0),(1.0,1.0,1.0,1.0 ; 1.0)] \varnothing \\
& \{[(1.0,1.0,1.0,1.0 ; 1.0),(1.0,1.0,1.0,1.0 ; 1.0)] \oplus \\
& {[(0.4025,0.4525,0.5375,0.5675 ; 0.5),(0.32,0.41,0.58,0.65 ; 1.0)] \oplus } \\
& {[(0.65,0.6725,0.7575,0.79 ; 0.5),(0.58,0.63,0.80,0.86 ; 1.0)] \oplus } \\
& {[(0.65,0.6725,0.7575,0.79 ; 0.5),(0.58,0.63,0.80,0.86 ; 1.0)]\}=}
\end{aligned}
$$




$$
\begin{aligned}
& {[(1.0,1.0,1.0,1.0 ; 1.0),(1.0,1.0,1.0,1.0 ; 1.0)] \varnothing } \\
& {[(2.7025,2.7975,3.0525,3.1475 ; 0.5),(2.48,2.67,3.18,3.37 ; 1.0)]=} \\
& {[(0.3177,0.3276,0.3575,0.3700 ; 0.5),(0.2967,0.3145,0.3745,0.4032 ; 1.0)], } \\
& \tilde{\tilde{\omega}}_{2}=[(0.1489,0.1618,0.1761,0.1803 ; 0.5),(0.1290,0.1536,0.1824,0.1929 ; 1.0)], \\
& \tilde{\tilde{\omega}}_{3}=[(0.2404,0.2405,0.2482,0.2510 ; 0.5),(0.2339,0.2360,0.2516,0.2552 ; 1.0)], \\
& \tilde{\tilde{\omega}}_{4}=[(0.2404,0.2405,0.2482,0.2510 ; 0.5),(0.2339,0.2360,0.2516,0.2552 ; 1.0)] .
\end{aligned}
$$

\begin{tabular}{|c|c|}
\hline Client & Situation description \\
\hline$C_{1}$ & $\begin{array}{l}\text { A manufactory corporation that has several affiliated companies. } \\
\text { Financial statements of affiliated companies now are audited by other CPA firms. } \\
\text { It produces consumer electronics. } \\
\text { The audit field work is estimated to take two weeks at interim audit and three weeks for } \\
\text { final audit. } \\
\text { It is going to be an initial public offering after two years. } \\
\text { The annual audit fee it could afford is about NTD } \$ 800,000 \text {. } \\
\text { In the first half of this year, approval for } C_{1} \text { 's investment in expanding plants was denied } \\
\text { by Environmental Impact Assessment (EIA) Committee. } \\
\text { The annual earnings in latest three years were approximately NTD } \$ 3 \text { per share. }\end{array}$ \\
\hline $\mathrm{C}_{2}$ & $\begin{array}{l}\text { A company in the textile industry. } \\
\text { The company was devoted to increasing its positive environmental impact in recent } \\
\text { years, which was reported by newspapers several times. } \\
C_{2} \text { is not a listed company, and the annual audit fee it could afford is about NTD } \\
\$ 600,000 \text {. } \\
\text { The annual earnings in latest three years were around NTD } \$ 1 \text { to } \$ 2 \text { per share. }\end{array}$ \\
\hline $\mathrm{C}_{3}$ & $\begin{array}{l}\text { A corporation that produces adult diapers. } \\
\text { The public always has a positive image of the corporate social responsibility of } C_{3} \\
\text { because of activities it devoted to public welfare. } \\
\text { The annual earnings in the latest three years were around NTD } \$ 3 \text { to } \$ 5 \text { per share. } \\
C_{3} \text { is not a listed company and the annual audit fee it could afford is about NTD } \\
\$ 400,000 \text {. }\end{array}$ \\
\hline $\mathrm{C}_{4}$ & $\begin{array}{l}\text { A corporation in the light emitting diode (LED) industry. } \\
C_{4} \text { just announced an intended merger with another LED company next year. } \\
\text { It is rare to see the corporation or its directors appearing in newspapers. } \\
C_{4} \text { is not a listed company, but the annual audit fee it could afford is about NTD } \\
\$ 800,000 \text {. } \\
\text { The annual earnings in latest three years were around NTD } \$ 0.5 \text { to } \$ 2 \text { per share. }\end{array}$ \\
\hline$C_{5}$ & $\begin{array}{l}\text { A company in the printed circuit board (PCB) industry. } \\
\text { The director was reported by a gossip magazine to be having an affair with his secretary. } \\
\text { Several employees died due to being overworked by the company. } \\
C_{5} \text { is considered to be a sweatshop. } \\
\text { The annual audit fee that } C_{5} \text { could afford is about NTD } \$ 1,200,000 \text { dollars. } \\
\text { The annual earnings in latest three years were around NTD } \$-0.5 \text { to } \$-1 \text { per share. }\end{array}$ \\
\hline
\end{tabular}

Table 3. Five potential clients' circumstances. 
Table 4. Linguistic values of the score for client acceptance evaluation items from DM\#1

\begin{tabular}{ccccc}
\hline $\begin{array}{c}\text { Attributes } \\
\text { Client }\end{array}$ & Benefit & Enterprise image & $\begin{array}{c}\text { Industrial } \\
\text { prospect }\end{array}$ & $\begin{array}{c}\text { Financial } \\
\text { status }\end{array}$ \\
\hline$C_{1}$ & Fairly high & Medium & Fairly high & High \\
$C_{2}$ & Medium & High & Medium & Medium \\
$C_{3}$ & Medium & High & High & Very high \\
$C_{4}$ & Medium & Medium & Fairly high & Medium \\
$C_{5}$ & Medium & Low & Medium & Low \\
\hline
\end{tabular}

Table 5. Linguistic values of the score for client acceptance evaluation items from DM\#2

\begin{tabular}{ccccc}
\hline $\begin{array}{c}\text { Attributes } \\
\text { Client }\end{array}$ & Benefit & $\begin{array}{c}\text { Enterprise } \\
\text { image }\end{array}$ & $\begin{array}{c}\text { Industrial } \\
\text { prospect }\end{array}$ & $\begin{array}{c}\text { Financial } \\
\text { status }\end{array}$ \\
\hline$C_{1}$ & Fairly low & Fairly low & Medium & Medium \\
$C_{2}$ & Medium & Fairly high & Medium & Fairly low \\
$C_{3}$ & Medium & High & High & High \\
$C_{4}$ & High & Medium & Medium & Fairly low \\
$C_{5}$ & Very high & Low & Fairly low & Low \\
\hline
\end{tabular}

Table 6. Linguistic values of the score for client acceptance evaluation items from DM\#3

\begin{tabular}{ccccc}
\hline $\begin{array}{c}\text { Attributes } \\
\text { Client }\end{array}$ & Benefit & $\begin{array}{c}\text { Enterprise } \\
\text { image }\end{array}$ & $\begin{array}{c}\text { Industrial } \\
\text { prospect }\end{array}$ & $\begin{array}{c}\text { Financial } \\
\text { status }\end{array}$ \\
\hline$C_{1}$ & Very high & Medium & Fairly high & High \\
$C_{2}$ & Fairly high & High & Medium & Fairly high \\
$C_{3}$ & Medium & High & Medium & High \\
$C_{4}$ & Very high & High & High & Fairly high \\
$C_{5}$ & Very high & Low & Medium & Fairly low \\
\hline
\end{tabular}

Step 2. Based on Eq. (10), we calculate the IVFN of the weighted scores of potential new client $C_{i}$ in the attribute $A_{j}$ under DM1's opinion as follows:

$$
\begin{aligned}
D M_{1}: & \\
\tilde{\tilde{C}}_{11}= & \tilde{\tilde{C}}_{11} \otimes \tilde{\tilde{\omega}}_{1}=[(0.65,0.6725,0.7575,0.79 ; 0.5),(0.58,0.63,0.8,0.86 ; 1.0)] \otimes \\
& {[(0.3177,0.3276,0.3575,0.3700 ; 0.5),(0.2967,0.3145,0.3745,0.4032 ; 1.0)]=} \\
& {[(0.2065,0.2203,0.2708,0.2923 ; 0.5),(0.1721,0.1981,0.2996,0.3468 ; 1.0)], } \\
\tilde{\tilde{C}}_{12}= & {[(0.0599,0.0732,0.0946,0.1023 ; 0.5),(0.0413,0.0629,0.1058,0.1254 ; 1.0)], } \\
\tilde{\tilde{C}}_{13}= & {[(0.1562,0.1617,0.1880,0.1983 ; 0.5),(0.1356,0.1486,0.2012,0.2195 ; 1.0)], } \\
\tilde{\tilde{C}}_{14}= & {[(0.1881,0.1960,0.2196,0.2278 ; 0.5),(0.1684,0.1840,0.2314,0.2475 ; 1.0)] . }
\end{aligned}
$$


Step 3. Based on Eqs (11)-(19), we use the proposed similarity measure to evaluate the degrees of similarity between the IVFNs of potential client $\tilde{\tilde{C}}$ and the outcome of the positive ideal solution $\tilde{\tilde{C}}^{*}$, respectively. The results are as follows:

$$
\begin{aligned}
& D M_{1}: \\
& S\left(\tilde{\tilde{C}}_{1}, \tilde{\tilde{C}}^{\star}\right)=0.8978 ; S\left(\tilde{\tilde{C}}_{2}, \tilde{\tilde{C}}^{\star}\right)=0.8362 ; S\left(\tilde{\tilde{C}}_{3}, \tilde{\tilde{C}}^{*}\right)=0.8652 ; \\
& S\left(\tilde{\tilde{C}}_{4}, \tilde{\tilde{C}}^{*}\right)=0.8511 ; S\left(\tilde{\tilde{C}}_{5}, \tilde{\tilde{C}}^{\star}\right)=0.7768 .
\end{aligned}
$$

Step 4. Based on Eqs (20)-(28), we use the proposed similarity measure to evaluate the degrees of similarity between the IVFNs of potential client $\tilde{\tilde{C}}_{i}$ and the outcome of the negative ideal solution $\tilde{\tilde{C}}-$. The results are as follows:

$$
\begin{aligned}
& D M_{1}: \\
& S\left(\tilde{\tilde{C}}_{1}, \tilde{\tilde{C}}_{-}\right)=0.7385 ; S\left(\tilde{\tilde{C}}_{2}, \tilde{\tilde{C}}_{-}\right)=0.7508 ; S\left(\tilde{\tilde{C}}_{3}, \tilde{\tilde{C}}_{-}\right)=0.7482 ; \\
& S\left(\tilde{\tilde{C}}_{4}, \tilde{\tilde{C}}_{-}\right)=0.7496 ; S\left(\tilde{\tilde{C}}_{5}, \tilde{\tilde{C}}_{-}\right)=0.7437
\end{aligned}
$$

Step 5. We calculate the relative closeness $R C_{i^{\star}}$ to the positive ideal solution (Eq. (29)).

$$
\begin{aligned}
& D M_{1}: \\
& R C_{1^{\star}}=0.5487 ; R C_{2^{\star}}=0.5269 ; R C_{3^{\star}}=0.5363 ; R C_{4^{\star}}=0.5317 ; R C_{5^{\star}}=0.5109 .
\end{aligned}
$$

Step 6. Finally, we rank the preference order. According to the descending order of the $R C_{i^{\star}}$ values, the preference order by DM1 is as follows:

$$
D M_{1}: C_{1} \succ C_{3} \succ C_{4} \succ C_{2} \succ C_{5} .
$$

The calculation results for each decision maker for each step are shown on Table A.1 in Appendix A.

\subsection{Comparative analysis and discussion}

We analyzed the results of the proposed method with the ranking directly provided by the decision makers. The comparison is summarized in Table 7 through Table 9.

Table 7. Comparison of results between the proposed method and manual ranking for DM1

\begin{tabular}{cccc}
\hline \multirow{2}{*}{ Client } & \multicolumn{2}{c}{ The proposed method } & \multirow{2}{*}{ Human ranking } \\
\cline { 2 - 3 } & $R C_{i^{*}}$ & Ranking & \\
\hline$C_{1}$ & 0.5487 & 1 & 1 \\
$C_{2}$ & 0.5269 & 4 & 4 \\
$C_{3}$ & 0.5363 & 2 & 3 \\
$C_{4}$ & 0.5317 & 3 & 2 \\
$C_{5}$ & 0.5109 & 5 & 5 \\
Spearman rank-order correlation coefficient & & 0.9 & \\
\hline
\end{tabular}


Table 8. Comparison of results between the proposed method and manual ranking for DM2

\begin{tabular}{cccc}
\hline \multirow{2}{*}{ Client } & \multicolumn{2}{c}{ The proposed method } & \multirow{2}{*}{ Human ranking } \\
\cline { 2 - 3 } & $R C_{i^{*}}$ & Ranking & 5 \\
\hline$C_{1}$ & 0.4647 & 5 & 4 \\
$C_{2}$ & 0.4850 & 4 & 2 \\
$C_{3}$ & 0.5037 & 1 & 1 \\
$C_{4}$ & 0.4912 & 2 & 3 \\
$C_{5}$ & 0.4861 & 3 & \\
\hline
\end{tabular}

Table 9. Comparison of results between the proposed method and manual ranking for DM3

\begin{tabular}{cccc}
\hline \multirow{2}{*}{ Client } & \multicolumn{2}{c}{ The proposed method } & \multirow{2}{*}{ Human ranking } \\
\cline { 2 - 3 } & $R C_{i^{*}}$ & Ranking & 2 \\
\hline$C_{1}$ & 0.5517 & 2 & 3 \\
$C_{2}$ & 0.5456 & 3 & 4 \\
$C_{3}$ & 0.5426 & 4 & 1 \\
$C_{4}$ & 0.5627 & 1 & 5 \\
$C_{5}$ & 0.5144 & 5 & \\
Spearman rank-order correlation coefficient & & 1.0 & \\
\hline
\end{tabular}

The Spearman rank-order correlation coefficient between the ranking results of the proposed method and ranking directly provided by the three decision makers are 0.9 to 1.0. This result indicates that the proposed method could help decision makers to evaluate potential clients before acceptance, especially in a situation where there are several potential clients but limited resources to provide service.

\section{Conclusions}

In this study, we used the TOPSIS approach to analyze client acceptance risk for an audit firm in the IVFN environment. Because the circumstances of an audit firm are always changing and are uncertain before potential clients are accepted, decision makers usually accept clients based on subjective judgments. Doing so will result in a risk of litigation for the audit firm or in a human resources problem. Using CAM, we suggest that audit firms establish fixed weights in advance for the criteria they consider using in linguistic terms. Therefore, no matter who performs the evaluation of potential new clients, the results will comply with a firm's policies. All the calculations could be written into a decision support system that will simplify the decision-making process. The only thing an audit firm has to do is to input partners' opinion in linguistic terms. CAM can also help audit firms that may have inadequate resources to do business with all those who seek their services to select clients from a pool of several potential clients. CAM takes on the role of problem analysis. It could provide the ranking of potential clients for decision makers to make client acceptance decisions. Determining the number of clients to accept depends on the resources available at a given audit firm.

Furthermore, rather than measuring the distance between the potential clients and the positive/negative ideal solution, we used the degree of similarity to replace distance in TOPSIS. The 
proposed method can overcome the drawbacks of the existing TOPSIS method by calculating the distances between IVFNs. Even when the same distance separates two IVFNs, they may have different shapes or directions, which can lead to nonintuitive results. Following the method presented by Chen and Sanguansat (2011), we applied the similarity measure between IVFNs in the decision-making process for client acceptance by audit firms. There are four criteria in the empirical study (benefit, enterprise image, industrial prospects and financial status) that are included in the weight of each factor. Considering communication patterns, we used linguistic terms to represent their corresponding IVFNs because IVFNs are more appropriate for expert opinions in some complex situations. CAM provides a useful means for audit firms to rank potential clients for acceptance in a variable, complex, and uncertain environment. Moreover, this method may also help audit firms to control the risks associated with client acceptance by establishing the weight of each criteria they consider. For further research, we suggest a discussion of in what circumstances a linguistic tool or a numerical tool would be more appropriate for problem analysis.

\section{Acknowledgments}

The authors are very grateful to the respected editor and the anonymous referees for their insightful and constructive comments, which helped to improve the overall quality of the paper. The authors are grateful to the grant funding support of Taiwan National Science Council (NSC 102-2410-H-182-013-MY3) during which the study was completed.

\section{References}

Baležentis, T.; Zeng, S. 2013. Group multi-criteria decision making based upon interval-valued fuzzy numbers: an extension of the MULTIMOORA method, Expert Systems with Applications 40(2): 543-550. http://dx.doi.org/10.1016/j.eswa.2012.07.066

Chang, S.-I.; Tsai, C.-F.; Shih, D.-H.; Hwang, C.-L. 2008. The development of audit detection risk assessment system: using the fuzzy theory and audit risk model, Expert Systems with Applications 35: 1053-1067. http://dx.doi.org/10.1016/j.eswa.2007.08.057

Chang, S. Y.; Yeh, T. Y. 2013. A two-echelon supply chain of a returnable product with fuzzy demand, Applied Mathematical Modelling 37(6): 4305-4315. http://dx.doi.org/10.1016/j.apm.2012.09.043

Chen, S. J. 2006. A new method for handling the similarity measure problems of interval-valued fuzzy numbers, in Proceedings of the Second International Conference on Natural Computation and the Third International Conference on Fuzzy Systems and Knowledge Discovery, 2006, Xian, China, 325-334.

Chen, S. J.; Chen, S. M. 2004. A new similarity measure between interval-valued fuzzy numbers, in Proceedings of the Joint Second International Conference of Soft Computing and Intelligent Systems and Fifth International Symposium on Advanced Intelligent Systems, 2004, Yokohama, Japan.

Chen, S. J.; Chen, S. M. 2008. Fuzzy risk analysis based on measures of similarity between interval-valued fuzzy numbers, Computers and Mathematics with Applications 55(8): 1670-1685. http://dx.doi.org/10.1016/j.camwa.2007.06.022

Chen, S. M.; Chen, J. H. 2009. Fuzzy risk analysis based on similarity measures between interval-valued fuzzy numbers and interval-valued fuzzy number arithmetic operators, Expert Systems with Applications 36: 6309-6317. http://dx.doi.org/10.1016/j.eswa.2008.08.017

Chen, S. M.; Sanguansat, K. 2011. Analyzing fuzzy risk based on similarity measures between interval-valued fuzzy numbers, Expert Systems with Applications 38(7): 8612-8621.

http://dx.doi.org/10.1016/j.eswa.2011.01.065 
Chen, T. Y. 2011. Optimistic and pessimistic decision making with dissonance reduction using interval-valued fuzzy sets, Information Sciences 181:479-502. http://dx.doi.org/10.1016/j.ins.2010.10.005

Chen, T. Y. 2012. Multiple criteria group decision-making with generalized interval-valued fuzzy numbers based on signed distances and incomplete weights, Applied Mathematical Modelling 36(7): 3029-3052. http://dx.doi.org/10.1016/j.apm.2011.09.080

Chen, T. Y.; Lai, H. L. 2011. A risk management method for enhancing patient safety based on interval-valued fuzzy numbers, African Journal of Business Management 5(30): 11925-11945.

Cimpoeru, S. S. 2011. Neural networks and their application in credit risk assessment. Evidence from the Romanian market, Technological and Economic Development of Economy 17(3): 519-534. http://dx.doi.org/10.3846/20294913.2011.606339

Craig, T. R. 1992. Should the client be accepted?, The CPA Journal 63(11): 64-66.

Farhadinia, B. 2014. Sensitivity analysis in interval-valued trapezoidal fuzzy number linear programming problems, Applied Mathematical Modelling 38(1): 50-62. http://dx.doi.org/10.1016/j.apm.2013.05.033

García, J.; Pazos, J.; Ríos, J.; Yagüe, J. 1992. Methodology of linguistics evaluation in risk situations using fuzzy techniques, Fuzzy Sets and Systems 48(2): 185-194.

http://dx.doi.org/10.1016/0165-0114(92)90332-X

Govindan, K.; Khodaverdi, R.; Jafarian, A. 2013. A fuzzy multi criteria approach for measuring sustainability performance of a supplier based on triple bottom line approach, Journal of Cleaner Production 47: 345-354. http://dx.doi.org/10.1016/j.jclepro.2012.04.014

Han, Z.; Liu, P. 2011. A fuzzy multi-attribute decision-making method under risk with unknown attribute weights, Technological and Economic Development of Economy 17(2): 246-258. http://dx.doi.org/10.3846/20294913.2011.580575

Hwang, C. L.; Yoon, K. 1981. Multiple attribute decision making: methods and applications. Berlin, Heidelberg, New York: Springer. http://dx.doi.org/10.1007/978-3-642-48318-9

Hwang, N.-C. R.; Chang, C. J. 2010. Litigation environment and auditors' decisions to accept clients' aggressive reporting, Journal of Accounting and Public Policy 29: 281-295. http://dx.doi.org/10.1016/j.jaccpubpol.2010.02.002

Idrus, A.; Nuruddin, M. F.; Rohman, M. A. 2011. Development of project cost contingency estimation model using risk analysis and fuzzy expert system, Expert Systems with Applications 38: 1501-1508. http://dx.doi.org/10.1016/j.eswa.2010.07.061

Jamal, K.; Sunder, S. 2011. Is mandated independence necessary for audit quality?, Accounting, Organizations and Society 36: 284-292. http://dx.doi.org/10.1016/j.aos.2011.03.001

Jensen, M. C.; Meckling, W. H. 1976. Theory of the firm: managerial behavior, agency costs and ownership structure, Journal of Financial Economics 3: 305-360. http://dx.doi.org/10.1016/0304-405X(76)90026-X

Johnson, V. E.; Khurana, J. K.; Reynolds, J. K. 2002. Audit-firm tenure and the quality of financial reports, Contemporary Accounting Research 19(4): 637-660. http://dx.doi.org/10.1506/LLTH-JXQV-8CEW-8MXD

Joshi, P. L.; Ajmi, J. A.; Bremser, W. G. 2009. A study of auditor-client relationships and problems in the Bahraini audit environment, Advances in Accounting, Incorporating Advances in International Accounting 25: 266-277.

Lai, H. L.; Chen, T. Y. 2011. A fuzzy risk-assessment method using a TOPSIS approach based on interval-valued fuzzy numbers, Journal of the Chinese Institute of Industrial Engineers 28(6): 467-484. http://dx.doi.org/10.1080/10170669.2011.613953

Leal-Ramirez, C.; Castillo, O.; Melin, P.; Rodriguez-Diaz, A. 2011. Simulation of the bird age-structured population growth based on an interval type-2 fuzzy cellular structure, Information Sciences 181: 519-535. http://dx.doi.org/10.1016/j.ins.2010.10.011

Mizumoto, M.; Tanaka, K. 1981. Fuzzy sets and type 2 under algebraic product and algebraic sum, Fuzzy Sets and Systems 5(3): 277-290. http://dx.doi.org/10.1016/0165-0114(81)90056-7 
Moizer, P.; Smith, L. H. 1998. Auditor liability: an insurable risk?, International Journal of Auditing 2(3): 197-214. http://dx.doi.org/10.1111/1099-1123.00040

Pappis, C. P.; Karacapilidis, N. I. 1995. Application of a similarity measure of fuzzy sets to fuzzy relational equations, Fuzzy Sets and Systems 75: 135-142. http://dx.doi.org/10.1016/0165-0114(95)00023-E

Sambuc, R. 1975. Fonctions U-floues, Application a l'aide au diagnostic en pathologie thyroidienne: $\mathrm{PhD}$ Thesis. University of Marseille, France.

Schmucker, K. J. 1984. Fuzzy sets, natural language computations and risk analysis. Rockville: Computer Science Press. 192 p.

Schneider, A. 2011. Is investment decision-making influenced by perceptions relating to auditors' client dependence and amount of audit fees?, Advances in Accounting, Incorporating Advances in International Accounting 27: 75-80.

Sepulveda, R.; Castillo, O.; Melin, P.; Rodriguez-Diaz, A.; Montiel, O. 2007. Experimental study of intelligent controllers under uncertainty using type-1 and type-2 fuzzy logic, Information Sciences 177(10): 2023-2048. http://dx.doi.org/10.1016/j.ins.2006.10.004

Steimann, F. 2001. On the use and usefulness of fuzzy sets in medical AI, Artificial Intelligence in Medicine 21: 131-137. http://dx.doi.org/10.1016/S0933-3657(00)00077-4

Tseng, M. L.; Chiu, A. S. F. 2013. Evaluating firm's green supply chain management in linguistic preferences, Journal of Cleaner Production 40: 22-31. http://dx.doi.org/10.1016/j.jclepro.2010.08.007

Wang, G.; Li, X. 1998. The applications of interval-valued fuzzy numbers and interval-distribution numbers, Fuzzy Sets and Systems 98(3): 331-335. http://dx.doi.org/10.1016/S0165-0114(96)00368-5

Wei, G.; Zhao, X.; Wang, H.; Lin, R. 2013. Fuzzy power aggregation operators and their application to multiple attribute group decision making, Technological and Economic Development of Economy 19(3): 377-396. http://dx.doi.org/10.3846/20294913.2013.821684

Wei, S. H.; Chen, S. M. 2009. Fuzzy risk analysis based on interval-valued fuzzy numbers, Expert Systems with Applications 36(2): 2285-2299. http://dx.doi.org/10.1016/j.eswa.2007.12.037

Wu, D.; Mendel, J. M. 2009. A comparative study of ranking methods, similarity measures and uncertainty measures for interval type-2 fuzzy sets, Information Sciences 179: 1169-1192. http://dx.doi.org/10.1016/j.ins.2008.12.010

Wu, D.; Wu, D. D.; Zhang, T.; Olson, D. L. 2013. Supply chain outsourcing risk using an integrated stochastic-fuzzy optimization approach, Information Sciences 235: 242-258. http://dx.doi.org/10.1016/j.ins.2013.02.002

Xu, Z.; Yager, R. R. 2008. Dynamic intuitionistic fuzzy multi-attribute decision making, International Journal of Approximate Reasoning 48: 246-262. http://dx.doi.org/10.1016/j.ijar.2007.08.008

Yu, D. 2013. Intuitionistic fuzzy prioritized operators and their application in multi-criteria group decision making, Technological and Economic Development of Economy 19(1): 1-21. http://dx.doi.org/10.3846/20294913.2012.762951

Zadeh, L. A. 1975. The concept of a linguistic variable and its application to approximate reasoning - I, Information Sciences 8(3): 199-249. http://dx.doi.org/10.1016/0020-0255(75)90036-5

Zadeh, L. A. 2005. Toward a generalized theory of uncertainty (GTU) - an outline, Information Sciences 172(1-2): 1-40. http://dx.doi.org/10.1016/j.ins.2005.01.017

Zhai, D.; Mendel, J. M. 2011. Uncertainty measures for general Type-2 fuzzy sets, Information Sciences 181: 503-518. http://dx.doi.org/10.1016/j.ins.2010.09.020

Zhang, H. Y.; Zhang, W. X. 2009. Hybrid monotonic inclusion measure and its use in measuring similarity and distance between fuzzy sets, Fuzzy Sets and Systems 160: 107-118. http://dx.doi.org/10.1016/j.fss.2008.06.002

Zhang, X.; Liu, P. 2010. Method for aggregating triangular fuzzy intuitionistic fuzzy information and its application to decision making, Technological and Economic Development of Economy 16(2): 280-290. http://dx.doi.org/10.3846/tede.2010.18 


\section{APPENDIX A}

Table A1. Results summary of each decision maker's opinion

\begin{tabular}{|c|c|c|c|}
\hline Items & $\mathrm{DM}_{1}$ & $\mathrm{DM}_{2}$ & $\mathrm{DM}_{3}$ \\
\hline$\tilde{\tilde{\omega}}_{1}$ & $\begin{array}{l}{[(0.3177,0.3276,0.3575} \\
0.3700 ; 0.5),(0.2967,0.3145 \\
0.3745,0.4032 ; 1.0)]\end{array}$ & $\begin{array}{l}{[(0.3273,0.3370,0.3646} \\
0.3752 ; 0.5),(0.3077,0.3247 \\
0.3802,0.4048 ; 1.0)]\end{array}$ & $\begin{array}{l}(0.2794,0.2818,0.2873, \\
0.2878 ; 0.5),(0.2740,0.2793, \\
0.2908,0.2924 ; 1.0)]\end{array}$ \\
\hline$\tilde{\tilde{\omega}}_{2}$ & $\begin{array}{l}{[(0.1489,0.1617,0.1761} \\
0.1803 ; 0.5),(0.1290,0.1535 \\
0.1824,0.1929 ; 1.0)]\end{array}$ & $\begin{array}{l}{[(0.2936,0.2970,0.2972,} \\
0.2982 ; 0.5),(0.2915,0.2966 \\
0.2985,0.2987 ; 1.0)]\end{array}$ & $\begin{array}{l}{[(0.2815,0.2839,0.2922,} \\
0.3033 ; 0.5),(0.2740,0.2793, \\
0.2967,0.3145 ; 1.0)]\end{array}$ \\
\hline$\tilde{\tilde{\omega}}_{3}$ & $\begin{array}{l}{[(0.2404,0.2405,0.2481} \\
0.2510 ; 0.5),(0.2339,0.2359 \\
0.2516,0.2552 ; 1.0)]\end{array}$ & $\begin{array}{l}{[(0.0872,0.0930,0.1095} \\
0.1170 ; 0.5),(0.0688,0.0836 \\
0.1169,0.1292 ; 1.0)]\end{array}$ & $\begin{array}{l}{[(0.1221,0.1322,0.1526,} \\
0.1597 ; 0.5),(0.1006,0.1217, \\
0.1620,0.1781 ; 1.0)]\end{array}$ \\
\hline$\tilde{\tilde{\omega}}_{4}$ & $\begin{array}{l}{[(0.2404,0.2405,0.2481} \\
0.2510 ; 0.5),(0.2339,0.2359 \\
0.2516,0.2552 ; 1.0)]\end{array}$ & $\begin{array}{l}{[(0.2439,0.2452,0.2553} \\
0.2586 ; 0.5),(0.2348,0.2395 \\
0.2597,0.2646 ; 1.0)]\end{array}$ & $\begin{array}{l}{[(0.2794,0.2818,0.2873,} \\
0.2878 ; 0.5),(0.2740,0.2793, \\
0.2908,0.2924 ; 1.0)]\end{array}$ \\
\hline$\tilde{\tilde{C}}_{11}$ & $\begin{array}{l}{[(0.2065,0.2203,0.2708,} \\
0.2923 ; 0.5),(0.17241,0.1981, \\
0.2996,0.3468 ; 1.0)]\end{array}$ & $\begin{array}{l}{[(0.0761,0.0859,0.1185} \\
0.1341 ; 0.5),(0.0523,0.0714 \\
0.1369,0.1700 ; 1.0)]\end{array}$ & $\begin{array}{l}{[(0.2647,0.2775,0.2852,} \\
0.2856 ; 0.5),(0.2548,0.2737, \\
0.2908,0.2924 ; 1.0)]\end{array}$ \\
\hline$\tilde{\tilde{C}}_{12}$ & $\begin{array}{l}{[(0.0599,0.0732,0.0946} \\
0.1023 ; 0.5),(0.0413,0.0629 \\
0.1058,0.1254 ; 1.0)]\end{array}$ & $\begin{array}{l}{[(0.0683,0.0757,0.0966,} \\
0.1066 ; 0.5),(0.0495,0.0652, \\
0.1074,0.1254 ; 1.0)]\end{array}$ & $\begin{array}{l}{[(0.1133,0.1285,0.1570,} \\
0.1721 ; 0.5),(0.0877,0.1145, \\
0.1721,0.2044 ; 1.0)] \\
\end{array}$ \\
\hline$\tilde{\tilde{C}}_{13}$ & $\begin{array}{l}{[(0.1562,0.1617,0.1880} \\
0.1983 ; 0.5),(0.1356,0.1486 \\
0.2012,0.2195 ; 1.0)]\end{array}$ & $\begin{array}{l}{[(0.0351,0.0421,0.0589,} \\
0.0664 ; 0.5),(0.0220,0.0343 \\
0.0678,0.0840 ; 1.0)]\end{array}$ & $\begin{array}{l}{[(0.0793,0.0889,0.1156,} \\
0.1262 ; 0.5),(0.0584,0.0766, \\
0.1296,0.1531 ; 1.0)]\end{array}$ \\
\hline$\tilde{\tilde{C}}_{14}$ & $\begin{array}{l}{[(0.1881,0.1960,0.2196,} \\
0.2278 ; 0.5),(0.1684,0.1840 \\
0.2314,0.2475 ; 1.0)]\end{array}$ & $\begin{array}{l}{[(0.0982,0.1109,0.1372,} \\
0.1467 ; 0.5),(0.0751,0.0982, \\
0.1506,0.1720 ; 1.0)]\end{array}$ & $\begin{array}{l}{[(0.2186,0.2296,0.2543,} \\
0.2612 ; 0.5),(0.1973,0.2179, \\
0.2675,0.2837 ; 1.0)]\end{array}$ \\
\hline$\tilde{\tilde{C}}_{21}$ & $\begin{array}{l}{[(0.1279,0.1482,0.1921,} \\
0.2100 ; 0.5),(0.0950,0.1289 \\
0.2172,0.2621 ; 1.0)]\end{array}$ & $\begin{array}{l}{[(0.1317,0.1525,0.1960,} \\
0.2129 ; 0.5),(0.0985,0.1331, \\
0.2205,0.2631 ; 1.0)]\end{array}$ & $\begin{array}{l}{[(0.1816,0.1895,0.2176,} \\
0.2274 ; 0.5),(0.1589,0.1760, \\
0.2326,0.2515 ; 1.0)]\end{array}$ \\
\hline$\tilde{\tilde{C}}_{22}$ & $\begin{array}{l}{[(0.1165,0.1318,0.1558} \\
0.1636 ; 0.5),(0.0929,0.1198 \\
0.1678,0.1871 ; 1.0)]\end{array}$ & $\begin{array}{l}{[(0.1908,0.1998,0.2251,} \\
0.2356 ; 0.5),(0.1691,0.1868, \\
0.2388,0.2569 ; 1.0)]\end{array}$ & $\begin{array}{l}{[(0.2203,0.2314,0.2586,} \\
0.2752 ; 0.5),(0.1973,0.2179, \\
0.2730,0.3050 ; 1.0)]\end{array}$ \\
\hline$\tilde{\tilde{C}}_{23}$ & $\begin{array}{l}{[(0.0967,0.1088,0.1334} \\
0.1424 ; 0.5),(0.0748,0.0967 \\
0.1459,0.1659 ; 1.0)]\end{array}$ & $\begin{array}{l}{[(0.0351,0.0421,0.0589} \\
0.0664 ; 0.5),(0.0220,0.0343 \\
0.0678,0.0840 ; 1.0)]\end{array}$ & $\begin{array}{l}{[(0.0491,0.0598,0.0820,} \\
0.0906 ; 0.5),(0.0322,0.0499, \\
0.0940,0.1157 ; 1.0)]\end{array}$ \\
\hline$\tilde{\tilde{C}}_{24}$ & $\begin{array}{l}{[(0.0967,0.1088,0.1334} \\
0.1424 ; 0.5),(0.0748,0.0967 \\
0.1459,0.1659 ; 1.0)]\end{array}$ & $\begin{array}{l}{[(0.0567,0.0625,0.0830} \\
0.0924 ; 0.5),(0.0399,0.0527 \\
0.0935,0.1111 ; 1.0)]\end{array}$ & $\begin{array}{l}{[(0.1816,0.1895,0.2176,} \\
0.2274 ; 0.5),(0.1589,0.1760, \\
0.2326,0.2515 ; 1.0)]\end{array}$ \\
\hline$\tilde{\tilde{C}}_{31}$ & $\begin{array}{l}{[(0.1279,0.1482,0.1921} \\
0.2100 ; 0.5),(0.0950,0.1289 \\
0.2172,0.2621 ; 1.0)]\end{array}$ & $\begin{array}{l}{[(0.1317,0.1525,0.1960,} \\
0.2129 ; 0.5),(0.0985,0.1331, \\
0.2205,0.2631 ; 1.0)]\end{array}$ & $\begin{array}{l}{[(0.1124,0.1275,0.1544,} \\
0.1633 ; 0.5),(0.0877,0.1145, \\
0.1687,0.1901 ; 1.0)]\end{array}$ \\
\hline$\tilde{\tilde{C}}_{32}$ & $\begin{array}{l}{[(0.1165,0.1318,0.1558} \\
0.1636 ; 0.5),(0.0929,0.1198 \\
0.1678,0.1871 ; 1.0)]\end{array}$ & $\begin{array}{l}{[(0.2297,0.2421,0.2630,} \\
0.2706 ; 0.5),(0.2099,0.2313 \\
0.2746,0.2897 ; 1.0)]\end{array}$ & $\begin{array}{l}{[(0.2203,0.2314,0.2586,} \\
0.2752 ; 0.5),(0.1973,0.2179, \\
0.2730,0.3050 ; 1.0)]\end{array}$ \\
\hline$\tilde{\tilde{C}}_{33}$ & $\begin{array}{l}{[(0.1881,0.1960,0.2196} \\
0.2278 ; 0.5),(0.1684,0.1840 \\
0.2314,0.2475 ; 1.0)]\end{array}$ & $\begin{array}{l}{[(0.0683,0.0758,0.0969,} \\
0.1062 ; 0.5),(0.0495,0.0652, \\
0.1075,0.1253 ; 1.0)]\end{array}$ & $\begin{array}{l}{[(0.0491,0.0598,0.0820,} \\
0.0906 ; 0.5),(0.0322,0.0499, \\
0.0940,0.1157 ; 1.0)]\end{array}$ \\
\hline
\end{tabular}


Continued Table A1

\begin{tabular}{|c|c|c|c|}
\hline Items & $\mathrm{DM}_{1}$ & $\mathrm{DM}_{2}$ & $\mathrm{DM}_{3}$ \\
\hline$\tilde{\tilde{C}}_{34}$ & $\begin{array}{l}{[(0.2278,0.2369,0.2463,} \\
0.2491 ; 0.5),(0.2175,0.2312, \\
0.2516,0.2552 ; 1.0)]\end{array}$ & $\begin{array}{l}(0.1908,0.1998,0.2259 \\
0.2347 ; 0.5),(0.1691,0.1868 \\
0.2390,0.2567 ; 1.0)]\end{array}$ & $\begin{array}{l}(0.2186,0.2296,0.2543 \\
0.2612 ; 0.5),(0.1973,0.2179 \\
0.2675,0.2837 ; 1.0)]\end{array}$ \\
\hline$\tilde{\tilde{C}}_{41}$ & $\begin{array}{l}{[(0.1279,0.1482,0.1921,} \\
0.2100 ; 0.5),(0.0950,0.1289 \\
0.2172,0.2621 ; 1.0)]\end{array}$ & $\begin{array}{l}{[(0.2561,0.2746,0.3227} \\
0.3405 ; 0.5),(0.2215,0.2532 \\
0.3498,0.3927 ; 1.0)]\end{array}$ & $\begin{array}{l}{[(0.2647,0.2775,0.2852,} \\
0.2856 ; 0.5),(0.2548,0.2737 \\
0.2908,0.2924 ; 1.0)]\end{array}$ \\
\hline$\tilde{\tilde{C}}_{42}$ & $\begin{array}{l}{[(0.0599,0.0732,0.0946} \\
0.1023 ; 0.5),(0.0413,0.0629 \\
0.1058,0.1254 ; 1.0)]\end{array}$ & $\begin{array}{l}{[(0.1182,0.1344,0.1597,} \\
0.1692 ; 0.5),(0.0933,0.1216 \\
0.1731,0.1941 ; 1.0)]\end{array}$ & $\begin{array}{l}{[(0.2203,0.2314,0.2586} \\
0.2752 ; 0.5),(0.1973,0.2179 \\
0.2730,0.3050 ; 1.0)]\end{array}$ \\
\hline$\tilde{\tilde{C}}_{43}$ & $\begin{array}{l}{[(0.1562,0.1617,0.1880,} \\
0.1983 ; 0.5),(0.1356,0.1486 \\
0.2012,0.2195 ; 1.0)]\end{array}$ & $\begin{array}{l}{[(0.0351,0.0421,0.0589,} \\
0.0664 ; 0.5),(0.0220,0.0343, \\
0.0678,0.0840 ; 1.0)]\end{array}$ & $\begin{array}{l}{[(0.0955,0.1077,0.1350,} \\
0.1450 ; 0.5),(0.0724,0.0949, \\
0.1490,0.1727 ; 1.0)]\end{array}$ \\
\hline$\tilde{\tilde{C}}_{44}$ & $\begin{array}{l}{[(0.0967,0.1088,0.1334,} \\
0.1424 ; 0.5),(0.0748,0.0967 \\
0.1459,0.1659 ; 1.0)]\end{array}$ & $\begin{array}{l}{[(0.0567,0.0625,0.0830} \\
0.0924 ; 0.5),(0.0399,0.0527 \\
0.0935,0.1111 ; 1.0)]\end{array}$ & $\begin{array}{l}{[(0.1816,0.1895,0.2176,} \\
0.2274 ; 0.5),(0.1589,0.1760 \\
0.2326,0.2515 ; 1.0)]\end{array}$ \\
\hline$\tilde{\tilde{C}}_{51}$ & $\begin{array}{l}{[(0.1279,0.1482,0.1921,} \\
0.2100 ; 0.5),(0.0950,0.1289, \\
0.2172,0.2621 ; 1.0)]\end{array}$ & $\begin{array}{l}{[(0.3101,0.3319,0.3619,} \\
0.3724 ; 0.5),(0.2861,0.3182, \\
0.3802,0.4048 ; 1.0)]\end{array}$ & $\begin{array}{l}{[(0.2647,0.2775,0.2852,} \\
0.2856 ; 0.5),(0.2548,0.2737 \\
0.2908,0.2924 ; 1.0)]\end{array}$ \\
\hline$\tilde{\tilde{C}}_{52}$ & $\begin{array}{l}{[(0.0130,0.0194,0.0282,} \\
0.0329 ; 0.5),(0.0052,0.0153, \\
0.0328,0.0444 ; 1.0)]\end{array}$ & $\begin{array}{l}(0.0257,0.0356,0.0475 \\
0.0544 ; 0.5),(0.0117,0.0296 \\
0.0537,0.0687 ; 1.0)]\end{array}$ & $\begin{array}{l}(0.0246,0.0341,0.0467 \\
0.0553 ; 0.5),(0.0109,0.0279 \\
0.0534,0.0723 ; 1.0)]\end{array}$ \\
\hline$\tilde{\tilde{C}}_{53}$ & $\begin{array}{l}{[(0.0967,0.1088,0.1334,} \\
0.1424 ; 0.5),(0.0748,0.0967 \\
0.1459,0.1659 ; 1.0)]\end{array}$ & $\begin{array}{l}{[(0.0203,0.0237,0.0356,} \\
0.0418, ; 0.5),(0.0117,0.0184, \\
0.0421,0.0543 ; 1.0)]\end{array}$ & $\begin{array}{l}{[(0.0491,0.0598,0.0820,} \\
0.0906 ; 0.5),(0.0322,0.04988 \\
, 0.0940,0.1157 ; 1.0)]\end{array}$ \\
\hline$\tilde{\tilde{C}}_{54}$ & $\begin{array}{l}{[(0.0210,0.0289,0.0397} \\
0.0458 ; 0.5),(0.0093,0.0236 \\
0.0453,0.0587 ; 1.0)]\end{array}$ & $\begin{array}{l}{[(0.0213,0.0294,0.0408} \\
0.0472 ; 0.5),(0.0094,0.0239 \\
0.0467,0.0609 ; 1.0)]\end{array}$ & $\begin{array}{l}(0.0649,0.0718,0.0934, \\
0.1029 ; 0.5),(0.0466,0.0614 \\
0.1047,0.1228 ; 1.0)]\end{array}$ \\
\hline$S\left(\tilde{\tilde{C}}_{11}, \tilde{\tilde{C}}_{11}^{*}\right)$ & 0.8852 & 0.7480 & 0.9867 \\
\hline$S\left(\tilde{\tilde{C}}_{12}, \tilde{\tilde{C}}_{12}^{*}\right)$ & 0.9055 & 0.7538 & 0.7231 \\
\hline$S\left(\tilde{\tilde{C}}_{13}, \tilde{\tilde{C}}_{13}^{*}\right)$ & 0.8992 & 0.7512 & 0.9305 \\
\hline$S\left(\tilde{\tilde{C}}_{14}, \tilde{\tilde{C}}_{14}^{*}\right)$ & 0.9013 & 0.7600 & 0.9136 \\
\hline$S\left(\tilde{\tilde{C}}_{1}, \tilde{\tilde{C}}^{*}\right)$ & 0.8978 & 0.7532 & 0.8885 \\
\hline$S\left(\tilde{\tilde{C}}_{21}, \tilde{\tilde{C}}_{21}^{*}\right)$ & 0.7574 & 0.7599 & 0.8442 \\
\hline$S\left(\tilde{\tilde{C}}_{22}, \tilde{\tilde{C}}_{22}^{*}\right)$ & 0.9027 & 0.7544 & 0.9131 \\
\hline$S\left(\tilde{\tilde{C}}_{23}, \tilde{\tilde{C}}_{23}^{*}\right)$ & 0.8423 & 0.7512 & 0.8691 \\
\hline$S\left(\tilde{\tilde{C}}_{24}, \tilde{\tilde{C}}_{24}^{*}\right)$ & 0.8423 & 0.7612 & 0.8442 \\
\hline$S\left(\tilde{\tilde{C}}_{2}, \tilde{\tilde{C}}^{*}\right)$ & 0.8362 & 0.7567 & 0.8676 \\
\hline
\end{tabular}


Continued Table A1

\begin{tabular}{|c|c|c|c|}
\hline Items & $\mathrm{DM}_{1}$ & $\mathrm{DM}_{2}$ & $\mathrm{DM}_{3}$ \\
\hline$S\left(\tilde{\tilde{C}}_{31}, \tilde{\tilde{C}}_{31}^{*}\right)$ & 0.7574 & 0.7599 & 0.7273 \\
\hline$S\left(\tilde{\tilde{C}}_{32}, \tilde{\tilde{C}}_{32}^{*}\right)$ & 0.9027 & 0.7475 & 0.9131 \\
\hline$S\left(\tilde{\tilde{C}}_{33}, \tilde{\tilde{C}}_{33}^{*}\right)$ & 0.9013 & 0.7411 & 0.8691 \\
\hline$S\left(\tilde{\tilde{C}}_{34}, \tilde{\tilde{C}}_{34}^{*}\right)$ & 0.8996 & 0.7465 & 0.9136 \\
\hline$S\left(\tilde{\tilde{C}}_{3}, \tilde{\tilde{C}}^{*}\right)$ & 0.8652 & 0.7487 & 0.8558 \\
\hline$S\left(\tilde{\tilde{C}}_{41}, \tilde{\tilde{C}}_{41}^{*}\right)$ & 0.7574 & 0.7498 & 0.9867 \\
\hline$S\left(\tilde{\tilde{C}}_{42}, \tilde{\tilde{C}}_{42}^{*}\right)$ & 0.9055 & 0.7579 & 0.9131 \\
\hline$S\left(\tilde{\tilde{C}}_{43}, \tilde{\tilde{C}}_{43}^{*}\right)$ & 0.8992 & 0.7512 & 0.9653 \\
\hline$S\left(\tilde{\tilde{C}}_{44}, \tilde{\tilde{C}}_{44}^{*}\right)$ & 0.8423 & 0.7612 & 0.8442 \\
\hline$S\left(\tilde{\tilde{C}}_{4}, \tilde{\tilde{C}}^{*}\right)$ & 0.8511 & 0.7550 & 0.9273 \\
\hline$S\left(\tilde{\tilde{C}}_{51}, \tilde{\tilde{C}}_{51}^{*}\right)$ & 0.7574 & 0.7381 & 0.9867 \\
\hline$S\left(\tilde{\tilde{C}}_{52}, \tilde{\tilde{C}}_{52}^{*}\right)$ & 0.8159 & 0.7308 & 0.5543 \\
\hline$S\left(\tilde{\tilde{C}}_{53}, \tilde{\tilde{C}}_{53}^{\star}\right)$ & 0.8423 & 0.7552 & 0.8691 \\
\hline$S\left(\tilde{\tilde{C}}_{54}, \tilde{\tilde{C}}_{54}^{*}\right)$ & 0.6917 & 0.7584 & 0.6326 \\
\hline$S\left(\tilde{\tilde{C}}_{5}, \tilde{\tilde{C}}^{*}\right)$ & 0.7768 & 0.7456 & 0.7607 \\
\hline$S\left(\tilde{\tilde{C}}_{11}, \tilde{\tilde{C}}_{\overline{11}}\right)$ & 0.7008 & 0.8563 & 0.7126 \\
\hline$S\left(\tilde{\tilde{C}}_{12}, \tilde{\tilde{C}}_{\overline{12}}\right)$ & 0.7499 & 0.8831 & 0.7279 \\
\hline$S\left(\tilde{\tilde{C}}_{13}, \tilde{\tilde{C}}_{\overline{13}}\right)$ & 0.7536 & 0.8894 & 0.7259 \\
\hline$S\left(\tilde{\tilde{C}}_{14}, \tilde{\tilde{C}}_{\overline{14}}\right)$ & 0.7497 & 0.8426 & 0.7210 \\
\hline$S\left(\tilde{\tilde{C}}_{1}, \tilde{\tilde{C}}^{-}\right)$ & 0.7385 & 0.8678 & 0.7218 \\
\hline$S\left(\tilde{\tilde{C}}_{21}, \tilde{\tilde{C}}_{\overline{2} 1}\right)$ & 0.7411 & 0.7443 & 0.7274 \\
\hline$S\left(\tilde{\tilde{C}}_{22}, \tilde{\tilde{C}}_{\overline{22}}\right)$ & 0.7546 & 0.6937 & 0.7149 \\
\hline
\end{tabular}


Continued Table A1

\begin{tabular}{|c|c|c|c|}
\hline Items & $\mathrm{DM}_{1}$ & $\mathrm{DM}_{2}$ & $\mathrm{DM}_{3}$ \\
\hline$S\left(\tilde{\tilde{C}}_{23}, \tilde{\tilde{C}}_{\overline{2} 3}\right)$ & 0.7538 & 0.8894 & 0.7207 \\
\hline$S\left(\tilde{\tilde{C}}_{24}, \tilde{\tilde{C}}_{\overline{2} 4}\right)$ & 0.7538 & 0.8869 & 0.7274 \\
\hline$S\left(\tilde{\tilde{C}}_{2}, \tilde{\tilde{C}}_{-}\right)$ & 0.7508 & 0.8036 & 0.7226 \\
\hline$S\left(\tilde{\tilde{C}}_{31}, \tilde{\tilde{C}}_{\overline{31}}\right)$ & 0.7411 & 0.7443 & 0.7293 \\
\hline$S\left(\tilde{\tilde{C}}_{32}, \tilde{\tilde{C}}_{\overline{32}}\right)$ & 0.7546 & 0.6302 & 0.7149 \\
\hline$S\left(\tilde{\tilde{C}}_{33}, \tilde{\tilde{C}}_{\overline{3} 3}\right)$ & 0.7497 & 0.8832 & 0.7207 \\
\hline$S\left(\tilde{\tilde{C}}_{34}, \tilde{\tilde{C}}_{\overline{34}}\right)$ & 0.7474 & 0.6938 & 0.7210 \\
\hline$S\left(\tilde{\tilde{C}}_{3}, \tilde{\tilde{C}}^{-}\right)$ & 0.7482 & 0.7379 & 0.7215 \\
\hline$S\left(\tilde{\tilde{C}}_{41}, \tilde{\tilde{C}}_{\overline{41}}\right)$ & 0.7411 & 0.5445 & 0.7126 \\
\hline$S\left(\tilde{\tilde{C}}_{42}, \tilde{\tilde{C}}_{\overline{42}}\right)$ & 0.7499 & 0.8080 & 0.7149 \\
\hline$S\left(\tilde{\tilde{C}}_{43}, \tilde{\tilde{C}}_{\overline{43}}\right)$ & 0.7536 & 0.8894 & 0.7278 \\
\hline$S\left(\tilde{\tilde{C}}_{44}, \tilde{\tilde{C}}_{\overline{44}}\right)$ & 0.7538 & 0.8869 & 0.7274 \\
\hline$S\left(\tilde{\tilde{C}}_{4}, \tilde{\tilde{C}}^{-}\right)$ & 0.7496 & 0.7822 & 0.7207 \\
\hline$S\left(\tilde{\tilde{C}}_{51}, \tilde{\tilde{C}}_{\overline{51}}\right)$ & 0.7411 & 0.4831 & 0.7126 \\
\hline$S\left(\tilde{\tilde{C}}_{52}, \tilde{\tilde{C}}_{\overline{52}}\right)$ & 0.7386 & 0.8903 & 0.7147 \\
\hline$S\left(\tilde{\tilde{C}}_{53}, \tilde{\tilde{C}}_{\overline{53}}\right)$ & 0.7538 & 0.8898 & 0.7207 \\
\hline$S\left(\tilde{\tilde{C}}_{54}, \tilde{\tilde{C}}_{\overline{54}}\right)$ & 0.7414 & 0.8900 & 0.7241 \\
\hline$S\left(\tilde{\tilde{C}}_{5}, \tilde{\tilde{C}}^{-}\right)$ & 0.7437 & 0.7883 & 0.7180 \\
\hline$R C_{1^{*}}$ & 0.5487 & 0.4647 & 0.5517 \\
\hline$R C_{2^{*}}$ & 0.5269 & 0.4850 & 0.5456 \\
\hline$R C_{3^{*}}$ & 0.5363 & 0.5037 & 0.5426 \\
\hline$R C_{4^{*}}$ & 0.5317 & 0.4912 & 0.5627 \\
\hline$R C_{5^{*}}$ & 0.5109 & 0.4861 & 0.5144 \\
\hline
\end{tabular}


Hung-Lin LAI received her PhD degree from the Graduate Institute of Business and Administration of Chang Gung University, Taiwan in 2013. She received BS degree in Department of Accountancy from National Chung Hsing University, MS degree in Department of Accountancy from National Taipei University. After working for KPMG CPA firm in Taiwan for 11 years, she became a Lecturer in the Department of Information Management at Lee-Ming Institute of Technology, Taiwan in 2005. She turned out to be an Assistant Professor in 2013. Currently, she is an Assistant Professor in the Department of Accounting Information at Chihlee Institute of Technology. She has publications in some journals, including Journal of the Chinese Institute of Industrial Engineers and African Journal of Business Management. Her current research interests include multiple criteria decision making and fuzzy set theory.

Ting-Yu CHEN is currently a Professor of the Graduate Institute of Business and Management and the Department of Industrial and Business Management at Chang Gung University in Taiwan. She received her BSc degree in Transportation Engineering and Management, MSc degree in Civil Engineering, and PhD degree in Traffic and Transportation from National Chiao Tung University in Taiwan. She has published over 120 papers in peer-reviewed journals and 100 papers in conference proceedings. She has received a number of awards and grants. She is an Honorary Member of the Phi Tau Phi Scholastic Honor Society of Taiwan. Her current research interests include multiple criteria decision analysis, fuzzy decision analysis and methods, consumer decision making, and consumer marketing applications. 